The following is the supplement data to the article:

\title{
Evaluation of Non-Target Long-term LC-HRMS Time Series Data using Multivariate Statistical Approaches
}

Kirsten Purschke ${ }^{a, b}$, Maryam Vosough ${ }^{\mathrm{c}, ~}{ }^{*}$, Juri Leonhardt ${ }^{\mathrm{d}}$, Markus Webera, Torsten C. Schmidt ${ }^{\mathrm{b}, \mathrm{e}, *}$

a Environmental Analysis, Currenta GmbH \& Co. OHG, CHEMPARK BLG Q18, D-51368 Leverkusen, Germany

b Instrumental Analytical Chemistry (IAC) and Centre for Water and Environmental Research (ZWU), University of Duisburg-Essen, Universitaetsstrasse 5, D-45141 Essen, Germany

c Department of Clean Technologies, Chemistry and chemical engineering research centre of Iran (CCERCI), Tehran, Iran, P.O. Box 14335-186 Tehran, Iran

d Production Analytics, Currenta GmbH \& Co. OHG, CHEMPARK BLG B562, D-41538 Dormagen, Germany

e IWW Zentrum Wasser, Moritzstrasse 26, 45476 Mülheim an der Ruhr, Germany

*Corresponding author e-mails: Maryam Vosough <vosough@ccerci.ac.ir> and Torsten C. Schmidt: $<$ torsten.schmidt@uni-due.de> 


\section{Table of Contents}

1 DESCRIPTION OF CHEMOMETRIC METHODS ................................................ 3

1.1 PCA for Exploratory Time Trend Assessment .......................................... 3

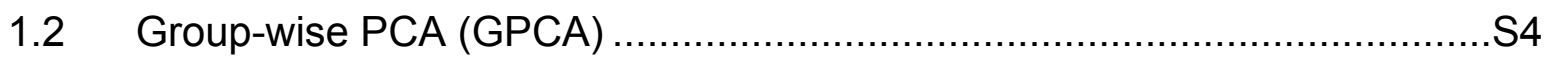

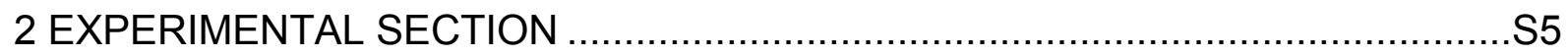

2.1 Datasets, Target Compounds and Internal Standards ....................................5 5

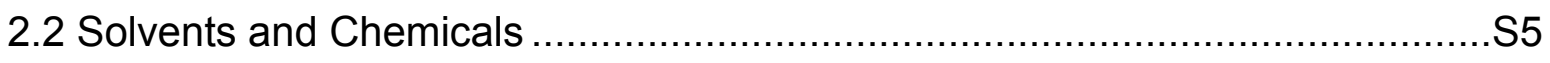

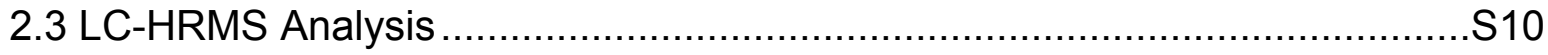

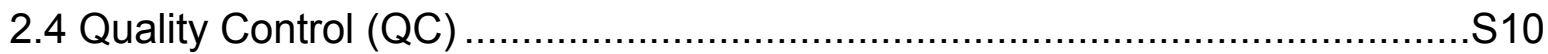

2.5 The Fusion of Target Data for Constructing Data Matrix of Both Ionisation Modes

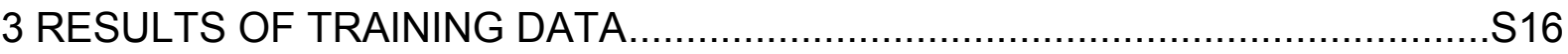

3.1 Overview of Detection of ISTD in Training Data Set .................................... 16

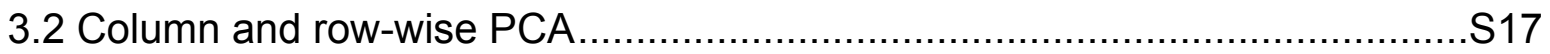

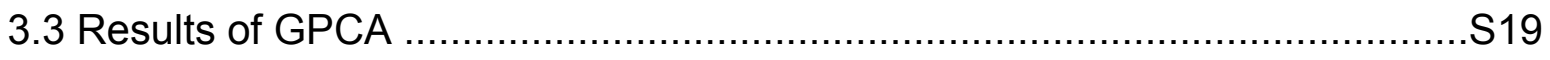

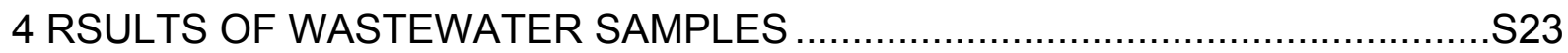

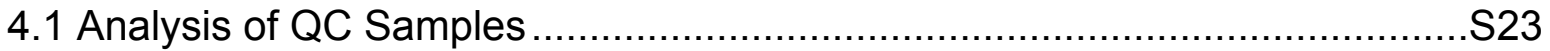

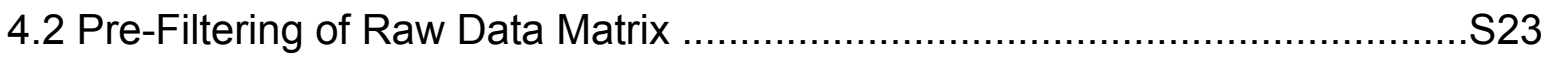

4.3 Results of Prioritising Samples in Time Series by Row-wise PCA ….............S27

4.4 GPCA Results and Prioritised Features Responsible for Trend Phenomena using

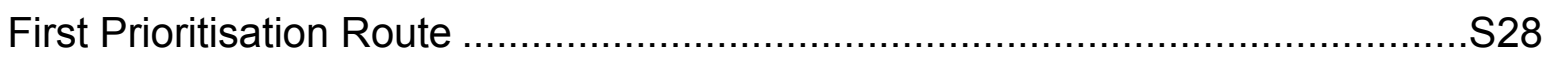

4.5 GPCA Results of Second Prioritisation Route ...........................................33

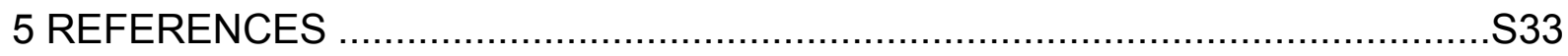




\section{DESCRIPTION OF CHEMOMETRIC METHODS}

\subsection{PCA for Exploratory Time Trend Assessment}

Applying PCA to data matrices (X) with $\mathrm{M}$ observations (time points) and $\mathrm{N}$ features, the subspace of maximum variance in the $\mathrm{N}$-dimensional feature space can be found. Principal components (PCs) which are linear transformations of original features are the eigenvectors of dispersion matrix of typically auto-scaled data. PCA model follows the expression:

$\mathbf{X}=\mathbf{T} \mathbf{P}^{\top}+\mathbf{E}$

where $\mathbf{T}$ is the $\mathrm{M} \times \mathrm{A}$ score matrix containing the projection of the objects onto the $\mathrm{A}$ number of $P C s, P$ is the $N \times A$ loading matrix and $\mathbf{E}$ is the $M \times N$ matrix of residual. A pair of statistics are commonly used to detect abnormal behaviour in a system: the Hotelling's $\mathrm{T}^{2}$ statistic (D-statistic), which is computed from the scores and is a measure of the variation of each object within the PCA model and Q-statistic which is the residual between an object and its projection into the model. These statistics for each object are defined as follows:

$D_{m}=t_{m} \times \Lambda^{-1} \times t_{m}{ }^{t}$

$Q_{m}=e_{m} \times e_{m}{ }^{t}$

where $t_{m}$ is the score vector in the $m^{\text {th }}$ row of $\mathbf{T}$ in equation $(I), \Lambda$ represents the covariance matrix of the scores and $e_{m}$ is the residual vector in the $m^{\text {th }}$ row of $E$ in equation (I). Change in the behaviour of objects can be detected by inspecting the score scatter plots, where scores of some objects are different from the rest of the data. However, the information regarding the objects and features (by PCA modelling of the transposed matrix) can be included in the Hotelling's $T^{2}$ statistic since it is calculated using the score values. A scatter plot of Hotelling's $\mathrm{T}^{2}$ statistic versus $\mathrm{Q}$-statistic is a preferable way of discerning any abnormal behaviour of objects based on exploratory PCA. Therefore, both statistics can be combined into a single selecting score, which is defined as Tscore ${ }^{1}$ for each observation as follows:

Tscore $_{m}=\alpha \times D_{m} / U C L^{D}+(1-\alpha) \times Q_{m} / U C L^{Q}$

where $\alpha$ is a weighting factor for this combination and set to the total variance of the model (in percent). $U C L^{D}$ and $U C L^{Q}$ are the upper control limits for the D-statistic and Q-statistic (at 95\% confidence level). This score was initially defined and implemented for intrusion detection in multivariate network monitoring studies ${ }^{2,3}$. In the present work, Tscore was evaluated for time trend exploration and feature prioritisation while a typical LC-HRMS data matrix and its transpose subjected to PCA in the different modelling process. 


\subsection{Group-wise PCA (GPCA)}

GPCA is a sparse variant of the PCA where an adaptation of PCA to a simplified group-wise model can be justified by the data set ${ }^{4}$. This method has been developed as one of the promising ways to overcome PCA disadvantages. The first disadvantage is that the unique variance for each variable cannot be discriminated from the shared variance among several variables, preventing the extraction of the hidden relationship between variables ${ }^{5}$. The second is that new latent variables (PCs) are usually a linear function of all variables, making the components difficult to interpret ${ }^{6}$. In GPCA, sparse solution is obtained in terms of groups of correlated variables. Here, every GPC contains non-zero loadings for a single group of correlated variables. So, the model and its interpretation will be considerably simplified.

The first step of this approach is the computation of association map between variables using MEDA (Missing-data for Exploratory Data analysis ${ }^{7}$ ) approach. Briefly, following PCA factorization on the matrix $\left(\mathbf{X}_{\mathrm{M} \times \mathrm{N}}\right)$, a new matrix $\tilde{\mathbf{Y}}_{j}$ is constructed for each variable of the matrix $\mathbf{X}$, so that all elements of this matrix are zero except for the $j$-th column, where it contains the $n$-th column of matrix $\mathbf{X}$. Then, using $\tilde{\mathbf{Y}}_{j}$ and $\mathrm{A}$ latent variable, the sores are estimated using missing data method of known data regression $(\mathrm{KDR})^{8}$. From the scores, the original data is reconstructed and then the estimation error is computed $(\hat{R})$. The variability of the estimation error is compared to that of the initial matrix $\mathbf{X}$ according to the index of goodness of prediction $\mathrm{q}_{\mathrm{j}, \mathrm{k}}^{2}$ for A latent variables:

$q_{\mathrm{A}, \mathrm{j}, \mathrm{k})}^{2}=1-\frac{\sum_{m=1}^{M}\left(\hat{\mathrm{R}}_{A,(m, k)}\right)^{2}}{\sum_{m=1}^{M}\left(\mathrm{X}_{m, k}\right)^{2}}, \quad \forall k \neq j$

The closer the $\mathrm{q}^{2}$ value to 1 the more the $\mathrm{j}$ and $\mathrm{k}$-th variables are related to each other. Finally, MEDA matrix is built from elements of $\mathrm{q}^{2} \mathrm{j,k}$ corresponding to each pair of variables for $A$ components in the model.

Then, $\mathrm{K}$ groups of correlated/associated variables are identified using the so-called group identification algorithm (GIA). For a correlation matrix of $\mathbf{Q}^{2}$ with the dimension of $N \times N$ and elements between -1 and 1 , a threshold $y$ (values between 0 to 1 ) can be defined in such a way that variables in $\mathbf{Q}^{2}$ matrix having the values larger than $y$ are selected for making a group $(G)$. y should be optimised for each specific data set by inspecting the visualisation of MEDA and the output of the GIA, interactively. Following the groups' definitions, the GPCA algorithm first computes $\mathrm{K}$ candidate and sparse loading vectors associated with the variables in the $\mathrm{k}^{\text {th }}$ group. Then, according to the selected model, the loading with the largest explained variance is extracted from matrix $\mathbf{X}$. further details regarding GPCA together with the algorithm is available in the literature ${ }^{4}$. 


\section{EXPERIMENTAL SECTION}

\subsection{Datasets, Target Compounds and Internal Standards}

Table S-1 - Table of data sets for data analysis method development and validation.

\begin{tabular}{|ccc|}
\hline & Validation dataset & Authentic dataset \\
\hline Samples & $\begin{array}{c}29 \text { Milli-Q }{ }^{\circledR} \text { samples spiked with 19 target } \\
\text { analytes (see Table S- 2) }\end{array}$ & 69 WWTP influent samples \\
Trends & $\begin{array}{c}\text { Artificially produced trends over the 29 spiked } \\
\text { samples }\end{array}$ & $\begin{array}{c}\text { Authentical trends over five months: 2018- } \\
11 \text { to 2019-03 }\end{array}$ \\
$\begin{array}{c}\text { Application } \\
\text { Aim }\end{array}$ & Method development & $\begin{array}{c}\text { Verification of developed data analysis for } \\
\text { authentic samples }\end{array}$ \\
& Determination of temporal trends in the NTS & Validation of advanced data analysis \\
\hline
\end{tabular}

\subsection{Solvents and Chemicals}

All solvents used in the present work were of LCMS grade. Methanol was from Honeywell Riedel-de-Haën ${ }^{T M}$ (Seelze, Germany). Ultra-pure water was used from a Milli-Q ${ }^{\circledR}\left(Q_{-}-P D^{\circledR}\right)$ water system from Merck KGaA (Darmstadt, Germany) and formic acid (99\%) was purchased from Fisher Chemical (Geel, Belgium). All internal standards (ISTD) and target analytes were of a quality grade suitable for trace analysis, purity $\geq 95 \%$. The corresponding chemical abstract service (CAS) numbers of used targets and ISTD are listed in the supporting information (see SI 2.1, Table S-2 and Table S-3). 
Table S- 2 - Spiked target analytes for training data set covering relevant (black) and non-relevant (grey) time trends ( $n=29$ ). All data are rounded data and refer to the $L C$-HRMS measurement. For the identification, a maximum mass error of \pm 2 ppm and a maximum retention time error of \pm 0.05 min were approved (*industrial chemical).

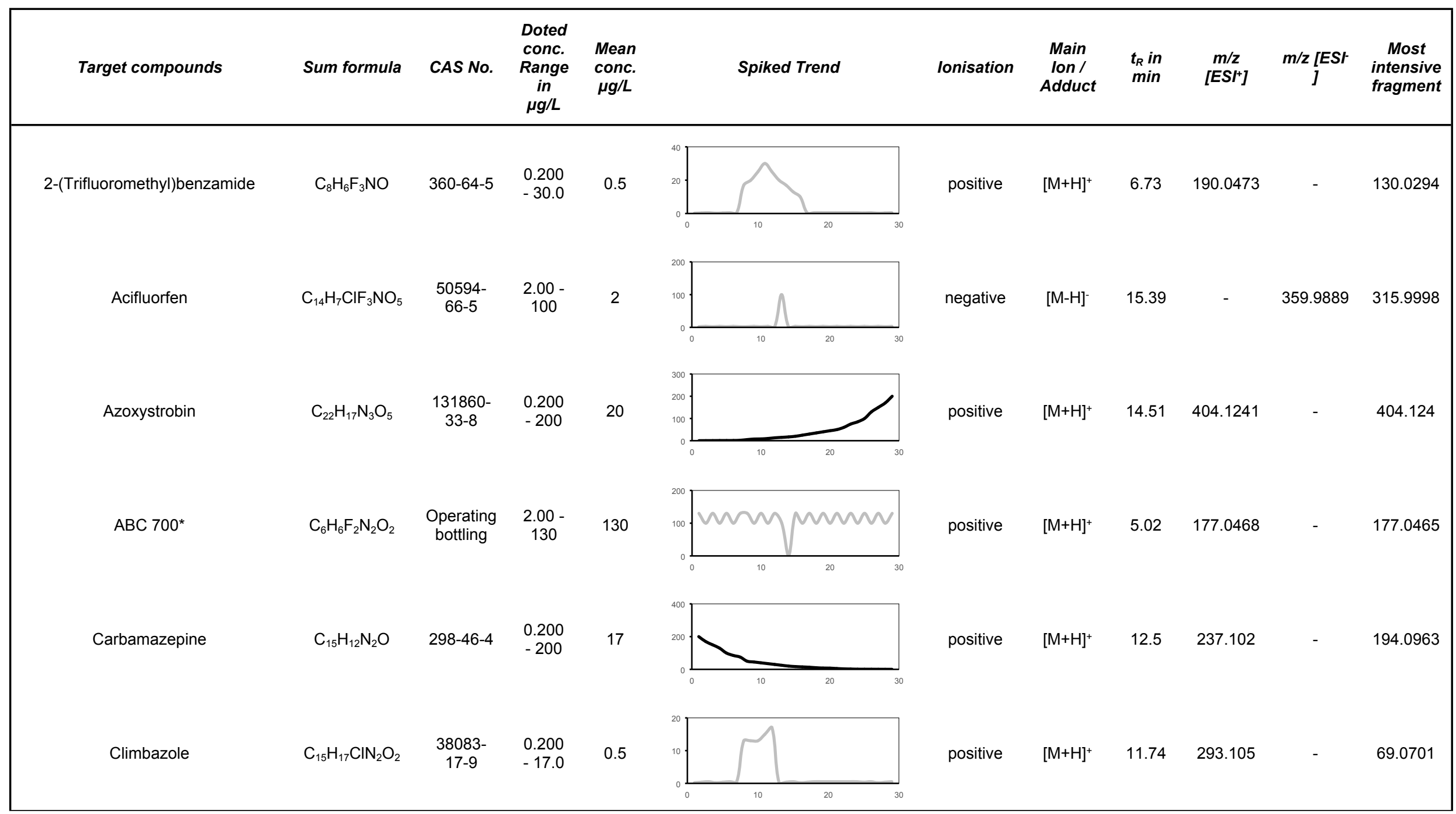




\section{Supporting Information}

Table S- 2 continued.

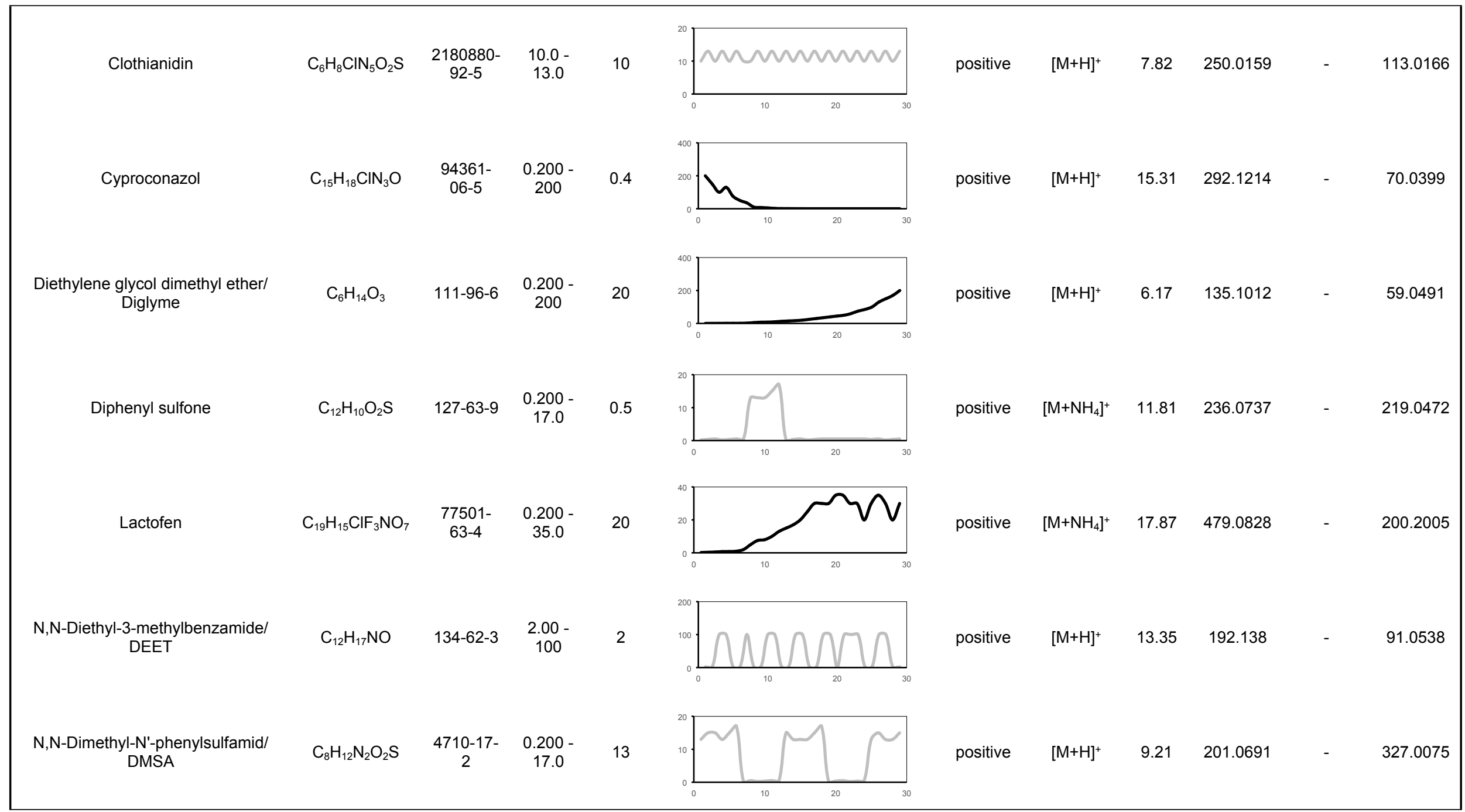




\section{Supporting Information}

Table S- 2 continued.

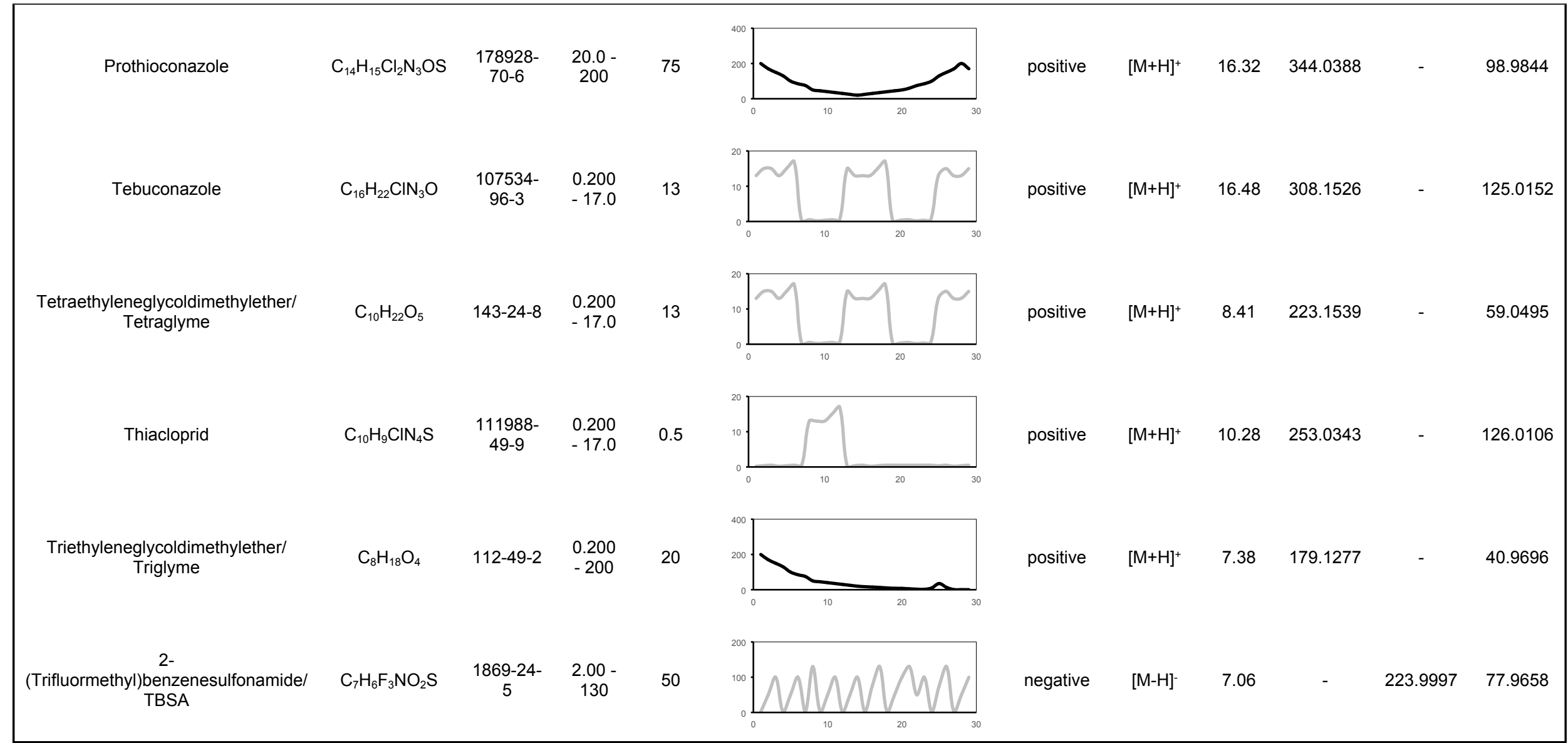


Table S- 3 - Table of used internal standards (ISTD). All data are rounded data and refer to the LC-HRMS measurement. For the identification, a maximum mass error of \pm 2 ppm and a maximum retention time error of \pm 0.05 min were approved. Fragment ions are listed in decreasing intensity from 1 to 5 . Pos $\triangleq$ positive ionisation mode, neg $\triangleq$ negative

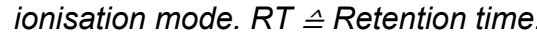

\begin{tabular}{|c|c|c|c|c|c|c|c|c|c|c|c|c|c|}
\hline Target compound & $\begin{array}{l}\text { Azoxy- } \\
\text { strobin- } \\
\text { D4 }\end{array}$ & $\begin{array}{c}\text { Bezafibrate- } \\
\text { D6 }\end{array}$ & $\begin{array}{l}\text { Chlor- } \\
\text { ampheni- } \\
\text { col-D5 }\end{array}$ & $\begin{array}{l}\text { Clothia- } \\
\text { nidin-D3 }\end{array}$ & $\begin{array}{c}\text { m-Toluic } \\
\text { acid } \\
\text { diethyla } \\
\text { mide-D7 } \\
\text { / DEET- } \\
\text { D7 }\end{array}$ & $\begin{array}{l}\text { Diclofenac- } \\
\text { D4 }\end{array}$ & Diuron-D6 & $\begin{array}{l}\text { Meco- } \\
\text { prop-D3 }\end{array}$ & $\begin{array}{c}\text { Mesotrione- } \\
\text { D4 }\end{array}$ & $\begin{array}{l}\text { Metsulfuron- } \\
\text { methyl-D3 }\end{array}$ & $\begin{array}{l}\text { Salicy } \\
\text { lic } \\
\text { Acid- } \\
\text { D4 }\end{array}$ & $\begin{array}{c}\text { Sulcotrione- } \\
\text { D4 }\end{array}$ & $\begin{array}{c}\text { Tebuco- } \\
\text { nazole- } \\
\text { D6 }\end{array}$ \\
\hline ESI mode & positive & pos / neg & negative & positive & positive & pos / neg & pos / neg & negative & pos / neg & pos / neg & negati & pos / neg & positive \\
\hline Main Ion / adduct & {$[\mathrm{M}+\mathrm{H}]^{+}$} & $\begin{array}{c}{[\mathrm{M}+\mathrm{H}]^{+} /[\mathrm{M}-} \\
\mathrm{H}]^{-}\end{array}$ & {$[\mathrm{M}-\mathrm{H}]^{-}$} & {$[\mathrm{M}+\mathrm{H}]^{+}$} & {$[\mathrm{M}+\mathrm{H}]^{+}$} & $\begin{array}{c}{[\mathrm{M}+\mathrm{H}]^{+} /[\mathrm{M}-} \\
\mathrm{H}]^{-}\end{array}$ & $\begin{array}{c}{[\mathrm{M}+\mathrm{H}]^{+} /[\mathrm{M}-} \\
\mathrm{H}]^{-}\end{array}$ & {$[\mathrm{M}-\mathrm{H}]^{-}$} & $\begin{array}{c}{[\mathrm{M}+\mathrm{H}]^{+} /[\mathrm{M}-} \\
\mathrm{H}]^{-}\end{array}$ & $\underset{\mathrm{H}]-}{[\mathrm{M}+\mathrm{H}]^{+} /[\mathrm{M}-}$ & {$[\mathrm{M}-\mathrm{H}]^{-}$} & $\begin{array}{c}{[\mathrm{M}+\mathrm{H}]^{+} /[\mathrm{M}-} \\
\mathrm{H}]^{-}\end{array}$ & {$[\mathrm{M}+\mathrm{H}]^{+}$} \\
\hline Sum formula & $\begin{array}{c}\mathrm{C}_{22} \mathrm{H}_{13} \mathrm{D}_{4} \\
\mathrm{~N}_{3} \mathrm{O}_{5}\end{array}$ & $\underset{4}{\mathrm{C}_{19} \mathrm{H}_{14} \mathrm{D}_{6} \mathrm{CINO}}$ & $\begin{array}{c}\mathrm{C}_{11} \mathrm{H}_{7} \mathrm{D}_{5} \mathrm{Cl}_{2} \\
\mathrm{~N}_{2} \mathrm{O}_{5}\end{array}$ & $\begin{array}{c}\mathrm{C}_{6} \mathrm{H}_{5} \mathrm{D}_{3} \mathrm{Cl} \\
\mathrm{N}_{5} \mathrm{O}_{2} \mathrm{~S}\end{array}$ & $\begin{array}{c}\mathrm{C}_{12} \mathrm{H}_{10} \mathrm{D}_{7} \\
\mathrm{NO}\end{array}$ & $\begin{array}{c}\mathrm{C}_{14} \mathrm{H}_{7} \mathrm{D}_{4} \mathrm{NO}_{2} \mathrm{Cl} \\
2\end{array}$ & $\mathrm{C}_{9} \mathrm{H}_{4} \mathrm{D}_{6} \mathrm{Cl}_{2} \mathrm{~N}_{2} \mathrm{O}$ & $\begin{array}{c}\mathrm{C}_{10} \mathrm{D}_{3} \mathrm{H}_{8} \\
\mathrm{ClO}_{3}\end{array}$ & $\mathrm{C}_{14} \mathrm{H}_{9} \mathrm{D}_{4} \mathrm{NO}_{7} \mathrm{~S}$ & $\begin{array}{c}\mathrm{C}_{14} \mathrm{H}_{12} \mathrm{D}_{3} \mathrm{~N}_{5} \mathrm{O}_{6} \\
\mathrm{~S}\end{array}$ & $\begin{array}{c}\mathrm{C}_{7} \mathrm{H}_{2} \mathrm{D} \\
{ }_{4} \mathrm{O}_{3}\end{array}$ & $\mathrm{C}_{14} \mathrm{H}_{9} \mathrm{D}_{4} \mathrm{ClO}_{5} \mathrm{~S}$ & $\begin{array}{c}\mathrm{C}_{16} \mathrm{H}_{16} \mathrm{D}_{6} \mathrm{C} \\
\mathrm{IN}_{3} \mathrm{O}\end{array}$ \\
\hline $\begin{array}{l}\text { Molecular mass in } \\
\mathrm{g}^{*} \mathrm{~mol}^{-1}\end{array}$ & 407.142 & 367.146 & 327.044 & 252.028 & 198.175 & 299.042 & 238.054 & 217.059 & 343.067 & 384.093 & $\begin{array}{c}142.0 \\
57\end{array}$ & 332.042 & 314.19 \\
\hline \multirow{2}{*}{$\begin{array}{c}\text { Expected mass in } \\
\mathrm{Da} \\
\text { Found at mass in } \\
\mathrm{Da}\end{array}$} & 408.149 & $\begin{array}{c}368.153 / \\
366.139\end{array}$ & 326.036 & 253.035 & 199.182 & $\begin{array}{c}300.049 / \\
298.035\end{array}$ & $\begin{array}{c}239.062 \text { I } \\
237.047\end{array}$ & 216.051 & $\begin{array}{c}344.074 / \\
342.059\end{array}$ & 385.1 & $\begin{array}{c}141.0 \\
5\end{array}$ & $\begin{array}{c}333.050 / \\
331.035\end{array}$ & 314.19 \\
\hline & 408.1487 & $\begin{array}{l}368.1527 / \\
366.1380\end{array}$ & 326.0362 & 253.0349 & 199.1822 & $\begin{array}{c}300.0489 / \\
298.0343\end{array}$ & $\begin{array}{c}239.0619 / \\
237.0473\end{array}$ & $\begin{array}{c}216.051 \\
2\end{array}$ & $\begin{array}{c}344.0737 / \\
342.0594\end{array}$ & $\begin{array}{c}385.1003 / \\
383.0859\end{array}$ & $\begin{array}{c}141.0 \\
494\end{array}$ & $\begin{array}{c}333.0493 / \\
331.0348\end{array}$ & 314.1903 \\
\hline Mass error in $\mathrm{mDa}$ & -0.467 & 0.657471 & -0.195 & 0.09 & -0.036 & 0.538793 & 1.140351 & -0.058 & $0.028 / 0.323$ & -2.279412 & -0.08 & 1.01581 & 0.212 \\
\hline Mass Error in ppm & -1.1 & 0.666667 & -0.6 & 0.4 & -0.2 & $0.4 /-0.8$ & $0.03 /-0.2$ & -0.3 & $0.1 / 0.9$ & -2.5 & -0.6 & 1 & 0.7 \\
\hline \multirow{2}{*}{$\begin{array}{c}\mathrm{RT} \text { in } \min \\
\text { Concentration in } \\
\mu \mathrm{g} / \mathrm{L}\end{array}$} & 14.47 & 14.09 & 7.93 & 10.28 & 13.3 & 15.7 & 13.73 & 14.73 & 10.55 & 11.73 & 8.33 & 11.82 & 16.43 \\
\hline & 0.025 & 25 & 25 & 25 & 1.25 & 25 & 25 & 25 & 25 & 25 & 25 & 0.25 & 1.25 \\
\hline \multirow{5}{*}{$\begin{array}{l}\text { Fragment } \\
\text { ions in } \mathrm{Da}\end{array}$} & 377.128 & $\begin{array}{c}138.9953 / \\
274.0642\end{array}$ & 157.066 & 126.011 & 126.094 & $\begin{array}{c}218.0674 / \\
34.9691\end{array}$ & $\begin{array}{l}78.0823 / \\
185.9522\end{array}$ & 216.033 & - & $\begin{array}{c}170.0759 / \\
142.0813\end{array}$ & $\begin{array}{c}97.05 \\
95\end{array}$ & - & 72.0537 \\
\hline & 349.134 & $\begin{array}{c}121.0655 / \\
154.0067\end{array}$ & 34.9695 & 90.034 & 98.0985 & $\begin{array}{c}254.0444 \text { / } \\
254.0446\end{array}$ & $\begin{array}{c}52.1028 / \\
34.9693\end{array}$ & 34.9694 & - & 135.044 & $\begin{array}{c}69.06 \\
48\end{array}$ & - & 279.232 \\
\hline & - & $\begin{array}{c}322.1486 / \\
41.9989\end{array}$ & 45.9936 & 253.0316 & 58.9428 & - & $\begin{array}{c}239.0608 / \\
237.0473\end{array}$ & $\begin{array}{c}197.048 \\
7\end{array}$ & - & 199.0081 & $\begin{array}{c}44.99 \\
81\end{array}$ & - & 95.0853 \\
\hline & - & $\begin{array}{l}276.0801 / / \\
90.0611\end{array}$ & 261.846 & - & 199.183 & - & - / 41.9985 & - & - & 209.983 & - & - & 314.182 \\
\hline & - & 166.128 & 326.034 & - & 44.0122 & - & - / 149.9749 & - & - & - & - & - & - \\
\hline
\end{tabular}




\subsection{LC-HRMS Analysis}

Table S-4 - LC-HRMS acquisition method.

\begin{tabular}{|c|c|}
\hline Parameter & Setting \\
\hline LC system & Agilent 1290 Infinity \\
\hline LC column & $\begin{array}{l}\text { Raptor }^{\mathrm{TM}} \text { Ultra Aqueous C18 analytical column }(100 \mathrm{~mm} \times 2.1 \mathrm{~mm}, 3.0 \mu \mathrm{m} \text { particle } \\
\text { size) linked to Restek }{ }^{\mathrm{TM}} \text { Trident cartridge }(10 \times 2.1 \mathrm{~mm}) \text { and a filter }(2 \mathrm{~mm}, 0.5 \mu \mathrm{m} \text {; } \\
\text { Restek GmbH Bad Homburg v. d. Höhe, Germany) }\end{array}$ \\
\hline Mobile Phase A & $0.1 \%$ formic acid in Milli- ${ }^{\circledR}$ \\
\hline Mobile Phase B & $0.1 \%$ formic acid in methanol \\
\hline Gradient $(\% \mathrm{~B})$ & $0.5 \min (0 \%$ B), $1.0 \min (10 \%$ B), $20 \min (90 \%$ B), $26 \min (90 \%$ B), $32 \min (0 \% B)$ \\
\hline Flow rate & $300 \mu \mathrm{L} \min ^{-1}$ \\
\hline Temperature & $55^{\circ} \mathrm{C}$ \\
\hline Sample injection volume & $5 \mu \mathrm{L}$ \\
\hline MS system & x500R qTOF (SCIEX) \\
\hline Ion source & Electrospray ion source Turbo $V^{\mathrm{TM}}$ \\
\hline MS mass range & $m / z 70-950$ \\
\hline Ion source gas 1 & 50 \\
\hline Ion source gas 2 & 70 \\
\hline Curtain gas & 40 \\
\hline Source temperature & $450{ }^{\circ} \mathrm{C}$ \\
\hline lonSpray voltage floating & $5500 \mathrm{~V} / \mathrm{-4500 \textrm {V }}$ \\
\hline Declustering potential & $80 \mathrm{~V}$ \\
\hline Collision energy & $7 \mathrm{~V}$ \\
\hline MS/MS Experiment & 10 data-dependent TOF-MS/MS scans \\
\hline MS/MS mass range & $m / z 30-700$ \\
\hline
\end{tabular}

\subsection{Quality Control (QC)}

To avoid false-positive or false-negative detection of trends resulting from instrumental drift, dependence on sample-composition matrix and batch-to-batch effects, an extensive batch-correction strategy, involving quality-control (QC) samples and internal standards (ISTDs) was employed for re-tuning the instrument and re-running the samples, whenever it was necessary. So, the stability/accuracy of the used LC-HRMS system during the whole experiment should be monitored by analysing quality control (QC) samples repeatedly throughout the sample sequence. The QC standard consisted of 70 target analytes (see Table S-5) with $100 \mu \mathrm{g} \mathrm{L}^{-1}$ concentration and an internal standard mixture of 13 isotope-labelled (deuterated) compounds (for concentrations see SI 2.1, Table S-3). The QC standard was run within each batch for both ionisation modes at the beginning and the end of each sequence. Furthermore, within each batch, a blank, consisting of $1 \mathrm{~mL}$ Milli- $\mathrm{Q}^{\circledR}$ and $0.1 \%$ formic acid 
spiked with ISTD, was measured to monitor for background contaminations. Furthermore, the (pre-)column of the LC system was changed depending on the peak shape of the analytes of the quality control. For internal and external standards, if the mass error exceeded $5 \mathrm{ppm}$, the instrument was re-tuned and samples were re-analysed. Additionally, after sample measurement, the ISTDs were analysed first. ISTDs should be categorised in the class of non-relevant trends (no trend), as they were spiked with the same concentration in each sample. An overview of detection, mass deviation and intensity ranges of the ISTDs is given in SI 3.1 (see Table S-6). As expected, the ISTDs showed no relevant time trend but showed a constant intensity over time.

\subsection{The Fusion of Target Data for Constructing Data Matrix of Both Ionisation Modes}

For target data, the analysis was performed on fused negative and positive ionisation data, facing two data sources, with a common retention time index, but different $\mathrm{m} / \mathrm{z}$ ratios and intensities. The data fusion enabled the discussion of all features in parallel. The total variance of the original data sources was different, due to the different performance of the two ionisation modes, which necessitates scaling of data for better comparability before analysing all features. Fusion is usually performed by using a scaling factor (the ratio of the first singular value of matrices). However, data scaling had no significant effect on the current results, due to the applied data pre-processing steps, which reduced both the skewness of the data and adapted variations of long-term data collection. For wastewater samples, positive and negative feature matrices were evaluated separately during the first route of data analysis, because of the amount of data and computational costs. The separate examination did not limit the compatibility to the validation data. 
Table S- $5-$ Table of analytes and ISTD used for quality control (QC). All data are rounded data. Fragment ions are listed in decreasing intensity from 1 to 5 . RT $\triangleq$ Retention time, $R \bumpeq$ Recovery.

\begin{tabular}{|c|c|c|c|c|c|c|c|c|c|c|c|}
\hline \multirow{2}{*}{ Target compounds } & \multirow{2}{*}{$\begin{array}{c}\text { Sum } \\
\text { formula }\end{array}$} & \multirow{2}{*}{ CAS No. } & \multirow{2}{*}{$\begin{array}{l}\text { Main lon } \\
\text { / Adduct }\end{array}$} & \multirow{2}{*}{$R T$ in min } & \multirow{2}{*}{$\begin{array}{c}\text { Expected } \\
\text { Mass in } \\
\text { Da }\end{array}$} & \multirow{2}{*}{$\begin{array}{c}\text { Found } \\
\text { Mass in } \\
\text { Da }\end{array}$} & \multicolumn{5}{|c|}{ Fragment ions in $\mathrm{Da}$} \\
\hline & & & & & & & 1 & 2 & 3 & 4 & 5 \\
\hline 1-(3'-Sulfophenyl)-3-methyl-5-aminopyrazole & $\mathrm{C}_{10} \mathrm{H}_{11} \mathrm{~N}_{3} \mathrm{O}_{3} \mathrm{~S}$ & $23646-86-8$ & {$[\mathrm{M}-\mathrm{H}]^{-}$} & 2.46 & 252.045 & 252.0446 & 79.9578 & 188.0831 & 107.0376 & - & - \\
\hline 1,5-Diaminonaphthalene & $\mathrm{C}_{10} \mathrm{H}_{10} \mathrm{~N}_{2}$ & $2243-62-1$ & {$[\mathrm{M}+\mathrm{H}]^{+}$} & 1.94 & 159.092 & 159.0915 & 115.0540 & 143.0740 & 159.0910 & 130.0650 & 89.0380 \\
\hline 1,6-Naphthalenedisulfonic acid & $\mathrm{C}_{10} \mathrm{H}_{8} \mathrm{O}_{6} \mathrm{~S}_{2}$ & $525-37-1$ & {$[\mathrm{M}-\mathrm{H}]^{-}$} & 2.03 & 286.969 & 286.9688 & 207.0123 & 143.0509 & 286.9693 & 79.9576 & - \\
\hline 1,7-Naphthalenedisulfonic acid & $\mathrm{C}_{10} \mathrm{H}_{8} \mathrm{O}_{6} \mathrm{~S}_{2}$ & $5724-16-3$ & {$[\mathrm{M}-\mathrm{H}]^{-}$} & 2.03 & 286.969 & 286.9687 & 207.0124 & 143.0509 & 286.9693 & 79.9576 & - \\
\hline 2-(n-Ethylanilino)ethanol & $\mathrm{C}_{10} \mathrm{H}_{15} \mathrm{NO}$ & $92-50-2$ & {$[\mathrm{M}+\mathrm{H}]^{+}$} & 2.68 & 166.123 & 166.1223 & 106.0650 & 120.0809 & 77.0387 & 166.1224 & 45.0333 \\
\hline 2-(Trifluormethyl)benzenesulfonamide/ TBSA & $\mathrm{C}_{7} \mathrm{H}_{6} \mathrm{~F}_{3} \mathrm{NO}_{2} \mathrm{~S}$ & $1869-24-5$ & {$[\mathrm{M}-\mathrm{H}]^{-}$} & 4.96 & 224.000 & 223.9997 & 77.9656 & 160.0384 & 100.0192 & 224.0000 & 145.0282 \\
\hline 2-(Trifluoromethyl)benzamide & $\mathrm{C}_{8} \mathrm{H}_{6} \mathrm{~F}_{3} \mathrm{NO}$ & $360-64-5$ & {$[\mathrm{M}+\mathrm{H}]^{+}$} & 5.32 & 190.047 & 190.0473 & 130.0300 & 102.0341 & 150.0358 & 170.0418 & 75.0234 \\
\hline 2,2'-(4-Methylphenylimino)diethanol & $\mathrm{C}_{11} \mathrm{H}_{17} \mathrm{NO}_{2}$ & 430227 & {$[\mathrm{M}+\mathrm{H}]^{+}$} & 3.25 & 196.133 & 196.1328 & 120.0806 & 134.0966 & 91.0543 & 196.1337 & 45.0336 \\
\hline 2,4,6-Triamino-1,3,5-triazine/ Melamine & $\mathrm{C}_{3} \mathrm{H}_{6} \mathrm{~N}_{6}$ & $108-78-1$ & {$[\mathrm{M}+\mathrm{H}]^{+}$} & 0.73 & 127.073 & 127.0724 & 85.0510 & 43.0289 & 68.0246 & 127.0727 & 110.0468 \\
\hline 2,4,6-Trimethoxy-1,3,5-triazine & $\mathrm{C}_{6} \mathrm{H}_{9} \mathrm{~N}_{3} \mathrm{O}_{3}$ & - & {$[\mathrm{M}+\mathrm{H}]^{+}$} & 6.77 & 172.072 & 172.0714 & 72.0444 & 58.0288 & 83.0241 & 140.0461 & - \\
\hline 2-Amino-4-methoxy-6-trifluormethyl-1,3,5-triazine/ AMTT & $\mathrm{C}_{5} \mathrm{H}_{5} \mathrm{~F}_{3} \mathrm{~N}_{4} \mathrm{O}$ & 1245970 & {$[\mathrm{M}+\mathrm{H}]^{+}$} & 5.90 & 195.049 & 195.0487 & 110.0222 & 43.0291 & 57.0447 & 57.0447 & 68.9951 \\
\hline 2-Chlorobenzoic acid & $\mathrm{C}_{7} \mathrm{H}_{5} \mathrm{O}_{2} \mathrm{Cl}$ & $118-91-2$ & {$[\mathrm{M}+\mathrm{H}]^{+}$} & 6.72 & 157.005 & 157.0050 & 77.0387 & 111.0000 & 138.9951 & 51.0233 & - \\
\hline 2-Naphthalenesulfonic acid & $\mathrm{C}_{10} \mathrm{H}_{8} \mathrm{SO}_{3}$ & $120-18-3$ & {$[\mathrm{M}-\mathrm{H}]^{-}$} & 5.29 & 207.012 & 207.0120 & 143.0505 & 207.0124 & 79.9575 & 115.0557 & - \\
\hline 3,5-Dihydroxybenzoic acid & $\mathrm{C}_{7} \mathrm{H}_{6} \mathrm{O}_{4}$ & $99-10-5$ & {$[\mathrm{M}-\mathrm{H}]^{-}$} & 2.66 & 153.093 & 153.0193 & 109.0299 & 41.0033 & 67.0190 & 65.0008 & 153.0244 \\
\hline 3-Aminobenzenesulfonic acid & $\mathrm{C}_{6} \mathrm{H}_{7} \mathrm{NO}_{3} \mathrm{~S}$ & $121-47-1$ & {$[\mathrm{M}-\mathrm{H}]^{-}$} & 0.71 & 172.007 & 172.0072 & 79.9572 & 172.0078 & 108.0455 & 40.0193 & - \\
\hline
\end{tabular}


Table S- 5 continued.

\begin{tabular}{|c|c|c|c|c|c|c|c|c|c|c|c|}
\hline 3-Hydroxy-2-nitrobenzoic acid & $\mathrm{C}_{7} \mathrm{H}_{5} \mathrm{NO}_{5}$ & $602-00-6$ & {$[\mathrm{M}-\mathrm{H}]^{-}$} & 3.44 & 182.009 & 182.0093 & 92.0266 & 45.9929 & 108.0235 & 138.0193 & - \\
\hline 3-Nitrophthalic acid & $\mathrm{C}_{8} \mathrm{H}_{5} \mathrm{NO}_{6}$ & $603-11-2$ & {$[\mathrm{M}-\mathrm{H}]^{-}$} & 2.66 & 210.004 & 210.0041 & 45.9933 & 122.0249 & 163.9552 & - & - \\
\hline 4-Aminobenzoic acid & $\mathrm{C}_{7} \mathrm{H}_{7} \mathrm{NO}_{2}$ & $150-13-0$ & {$[\mathrm{M}+\mathrm{H}]^{+}$} & 2.80 & 138.055 & 138.0549 & 77.0387 & 65.0386 & 92.0497 & 120.0449 & 51.0226 \\
\hline 4-Chlor-2-cyano-5-(4-methylphenyl)imidazole/ CCIM & $\mathrm{C}_{11} \mathrm{H}_{8} \mathrm{CIN}_{3}$ & $120118-14-1$ & {$[\mathrm{M}+\mathrm{H}]^{+}$} & 13.06 & 218.048 & 218.0478 & 183.0790 & 139.0310 & 130.0650 & 103.0550 & 118.0660 \\
\hline 4-Chlorobenzoic acid & $\mathrm{C}_{7} \mathrm{H}_{5} \mathrm{ClO}_{2}$ & $74-11-3$ & {$[\mathrm{M}-\mathrm{H}]^{-}$} & 9.77 & 154.991 & 154.9904 & 34.9692 & 111.0009 & 44.9978 & 154.9964 & - \\
\hline 4-Hydroxyphenylglycolic acid & $\mathrm{C}_{8} \mathrm{H}_{8} \mathrm{O}_{4}$ & $1198-84-1$ & {$[\mathrm{M}-\mathrm{H}]^{-}$} & 1.56 & 167.035 & 167.0350 & - & - & - & - & - \\
\hline 4-Sulphopthalic acid & $\mathrm{C}_{8} \mathrm{H}_{6} \mathrm{O}_{7} \mathrm{~S}$ & $89-08-07$ & {$[\mathrm{M}-\mathrm{H}]^{-}$} & 0.97 & 244.976 & 244.9767 & - & - & - & - & - \\
\hline 4-Toluenesulfonic acid & $\mathrm{C}_{7} \mathrm{H}_{8} \mathrm{O}_{3} \mathrm{~S}$ & $6192-52-5$ & {$[\mathrm{M}-\mathrm{H}]^{-}$} & 2.82 & 171.012 & 171.0121 & 79.9571 & 171.0120 & 107.0503 & - & - \\
\hline 6-Chloropyridine-2-carboxylic acid & $\mathrm{C}_{6} \mathrm{H}_{4} \mathrm{CINO}_{2}$ & $4684-94-0$ & {$[\mathrm{M}+\mathrm{H}]^{+}$} & 4.30 & 158.000 & 158.0002 & 111.9948 & 76.0183 & 139.9901 & 84.9840 & 75.0106 \\
\hline Acifluorfen & $\mathrm{C}_{14} \mathrm{H}_{7} \mathrm{ClF}_{3} \mathrm{NO}_{5}$ & $50594-66-5$ & {$[\mathrm{M}-\mathrm{H}]^{-}$} & 13.77 & 359.989 & 359.9890 & 194.9836 & 222.0303 & 286.0016 & 315.9991 & 45.9938 \\
\hline Azoxystrobin & $\mathrm{C}_{22} \mathrm{H}_{17} \mathrm{~N}_{3} \mathrm{O}_{5}$ & $131860-33-8$ & {$[\mathrm{M}+\mathrm{H}]^{+}$} & 13.07 & 404.124 & 404.1234 & 372.0970 & 344.1026 & 329.0792 & 345.1056 & 330.0820 \\
\hline $\mathrm{ABC} 700$ & $\mathrm{C}_{6} \mathrm{H}_{6} \mathrm{~F}_{2} \mathrm{~N}_{2} \mathrm{O}_{2}$ & - & {$[\mathrm{M}+\mathrm{H}]^{+}$} & 3.56 & 177.047 & 177.1234 & 137.0345 & 42.0344 & 109.0399 & 177.0489 & - \\
\hline Benzamideoxime & $\mathrm{C}_{7} \mathrm{H}_{8} \mathrm{~N}_{2} \mathrm{O}$ & $613-92-3$ & {$[\mathrm{M}+\mathrm{H}]^{+}$} & 1.78 & 137.071 & 137.0709 & 77.0389 & 104.0500 & 51.0233 & 137.0712 & 119.0610 \\
\hline Benzenesulfonic acid & $\mathrm{C}_{6} \mathrm{H}_{6} \mathrm{O}_{3} \mathrm{~S}$ & $98-11-3$ & {$[\mathrm{M}-\mathrm{H}]^{-}$} & 1.79 & 156.996 & 156.9963 & 79.9576 & 93.0347 & 156.9967 & - & - \\
\hline Benzoic acid & $\mathrm{C}_{7} \mathrm{H}_{6} \mathrm{O}_{2}$ & $65-85-0$ & {$[\mathrm{M}-\mathrm{H}]^{-}$} & 3.66 & 121.030 & 121.0296 & 77.0408 & 121.0266 & 92.0274 & - & - \\
\hline Carbamazepine & $\mathrm{C}_{15} \mathrm{H}_{12} \mathrm{~N}_{2} \mathrm{O}$ & $298-46-4$ & {$[\mathrm{M}+\mathrm{H}]^{+}$} & 10.84 & 237.102 & 237.1020 & 194.0958 & 179.0730 & 165.0700 & 152.0630 & - \\
\hline Chlorphenol (sum) & $\mathrm{C}_{6} \mathrm{H}_{5} \mathrm{ClO}$ & $95-57-8$ & {$[\mathrm{M}-\mathrm{H}]^{-}$} & 7.75 & 126.996 & 126.9955 & 34.9694 & 91.0184 & - & - & - \\
\hline Ciprofloxacin & $\mathrm{C}_{17} \mathrm{H}_{18} \mathrm{FN}_{3} \mathrm{O}_{3}$ & 85721-33-1 & {$[\mathrm{M}+\mathrm{H}]^{+}$} & 5.70 & 332.140 & 332.1400 & 314.1295 & 231.0570 & 332.1387 & 288.1498 & 98.9847 \\
\hline Climbazole & $\mathrm{C}_{15} \mathrm{H}_{17} \mathrm{ClN}_{2} \mathrm{O}_{2}$ & $38083-17-9$ & {$[\mathrm{M}+\mathrm{H}]^{+}$} & 10.14 & 293.105 & 293.1048 & 69.0699 & 197.0726 & 141.0104 & 225.0671 & - \\
\hline Clothianidin & $\mathrm{C}_{6} \mathrm{H}_{8} \mathrm{CIN}_{5} \mathrm{O}_{2} \mathrm{~S}$ & $\begin{array}{c}2180880-92- \\
5\end{array}$ & {$[\mathrm{M}+\mathrm{H}]^{+}$} & 6.15 & 250.016 & 250.0161 & 131.9673 & 113.0168 & 169.0547 & 110.0722 & 70.9959 \\
\hline Cyproconazol & $\mathrm{C}_{15} \mathrm{H}_{18} \mathrm{CIN}_{3} \mathrm{O}$ & $94361-06-5$ & {$[\mathrm{M}+\mathrm{H}]^{+}$} & 13.73 & 292.121 & 292.1209 & 70.0399 & 125.0152 & 138.9956 & - & - \\
\hline
\end{tabular}


Table S- 5 continued.

\begin{tabular}{|c|c|c|c|c|c|c|c|c|c|c|c|}
\hline Diethylene glycol dimethyl ether/ Diglyme & $\mathrm{C}_{6} \mathrm{H}_{14} \mathrm{O}_{3}$ & $111-96-6$ & {$[\mathrm{M}+\mathrm{H}]^{+}$} & 4.62 & 135.102 & 135.1015 & 59.0495 & 84.9597 & 116.9289 & - & - \\
\hline Diphenyl sulfone & $\mathrm{C}_{12} \mathrm{H}_{10} \mathrm{O}_{2} \mathrm{~S}$ & $127-63-9$ & {$\left[\mathrm{M}+\mathrm{NH}_{4}\right]^{+}$} & 10.15 & 236.074 & 236.0742 & 77.0390 & 219.0493 & 141.0004 & - & - \\
\hline Ditolyletherdisulfonic acid & $\mathrm{C}_{14} \mathrm{H}_{14} \mathrm{O}_{7} \mathrm{~S}_{2}$ & - & {$[\mathrm{M}-\mathrm{H}]^{-}$} & 3.97 & 357.011 & 357.0110 & 277.0550 & 185.9991 & 122.0378 & 79.9590 & - \\
\hline Diuron & $\mathrm{C}_{9} \mathrm{H}_{10} \mathrm{Cl}_{2} \mathrm{~N}_{2} \mathrm{O}$ & $330-54-1$ & {$[\mathrm{M}+\mathrm{H}]^{+}$} & 11.98 & 233.024 & 233.0241 & 72.0444 & 233.0238 & 159.9713 & 132.9614 & 187.9656 \\
\hline Fluopyram & $\mathrm{C}_{16} \mathrm{H}_{11} \mathrm{ClF}_{6} \mathrm{~N}_{2} \mathrm{O}$ & $\begin{array}{c}658066-35- \\
4\end{array}$ & {$[\mathrm{M}+\mathrm{H}]^{+}$} & 13.82 & 397.054 & 397.0533 & 173.0215 & 208.0140 & 397.0544 & 1445.0260 & 130.0300 \\
\hline Imidacloprid & $\mathrm{C}_{9} \mathrm{H}_{10} \mathrm{ClN}_{5} \mathrm{O}_{2}$ & $\begin{array}{c}138261-41- \\
3\end{array}$ & {$[\mathrm{M}+\mathrm{H}]^{+}$} & 6.70 & 256.060 & 256.0593 & 175.0978 & 209.0589 & 84.0541 & 128.0266 & - \\
\hline Ircadine & $\mathrm{C}_{12} \mathrm{H}_{23} \mathrm{NO}_{3}$ & $\begin{array}{c}119515-38- \\
7\end{array}$ & {$[\mathrm{M}+\mathrm{H}]^{+}$} & 12.12 & 230.175 & 230.1749 & 130.1223 & 95.0856 & 112.1122 & 84.0808 & 156.1025 \\
\hline Lactofen & $\mathrm{C}_{19} \mathrm{H}_{15} \mathrm{ClF}_{3} \mathrm{NO}_{7}$ & $77501-63-4$ & {$\left[\mathrm{M}+\mathrm{NH}_{4}\right]^{+}$} & 16.53 & 479.080 & 479.0823 & 344.9975 & 222.9776 & - & - & - \\
\hline Maleic acid & $\mathrm{C} 4 \mathrm{H} 4 \mathrm{O} 4$ & $110-16-7$ & {$[\mathrm{M}-\mathrm{H}]-$} & 1.10 & 115.004 & 115.0035 & - & - & - & - & - \\
\hline DEF $100^{*}$ & $\mathrm{C} 12 \mathrm{H} 8 \mathrm{~N} 2 \mathrm{OS}$ & - & {$[\mathrm{M}+\mathrm{H}]+$} & 15.65 & 229.043 & 229.0429 & 110.9904 & 82.9951 & - & - & - \\
\hline Morpholine & C4H9NO & $110-91-8$ & {$[\mathrm{M}+\mathrm{H}]+$} & 0.64 & 88.076 & 88.0754 & 45.0333 & 70.0654 & 42.0338 & 55.0415 & - \\
\hline Moxifloxacin & $\mathrm{C} 21 \mathrm{H} 24 \mathrm{FN} 3 \mathrm{O} 4$ & $\begin{array}{c}151096-09- \\
2\end{array}$ & {$[\mathrm{M}+\mathrm{H}]+$} & 7.70 & 402.182 & 402.1820 & 402.1824 & 384.1664 & 403.1892 & 364.1619 & 261.1060 \\
\hline $\mathrm{N}$-(M-Tolyl)-diethanolamine & $\mathrm{C} 11 \mathrm{H} 17 \mathrm{NO} 2$ & $91-99-6$ & {$[\mathrm{M}+\mathrm{H}]+$} & 3.72 & 196.133 & 169.1327 & 120.0807 & 134.0961 & 91.0542 & 45.0334 & - \\
\hline N,N-Diethyl-3-methylbenzamide/ DEET & $\mathrm{C} 12 \mathrm{H} 17 \mathrm{NO}$ & $134-62-3$ & {$[\mathrm{M}+\mathrm{H}]+$} & 11.67 & 192.138 & 192.1379 & 91.0538 & 119.0486 & 44.0130 & - & - \\
\hline N,N-Dimethyl-N'-phenylsulfamid/ DMSA & $\mathrm{C} 8 \mathrm{H} 12 \mathrm{~N} 2 \mathrm{O} 2 \mathrm{~S}$ & $4710-17-2$ & {$[\mathrm{M}+\mathrm{H}]+$} & 7.42 & 201.069 & 201.0693 & 92.0496 & 65.0389 & 137.1071 & - & - \\
\hline N-Allylthiourea & $\mathrm{C} 4 \mathrm{H} 8 \mathrm{~N} 2 \mathrm{~S}$ & $109-57-9$ & {$[\mathrm{M}+\mathrm{H}]+$} & 2.08 & 117.048 & 117.0481 & 40.9699 & 84.9589 & 58.0662 & - & - \\
\hline N-Nitrosomorpholine & $\mathrm{C} 4 \mathrm{H} 8 \mathrm{~N} 2 \mathrm{O} 2$ & $59-89-2$ & {$[\mathrm{M}+\mathrm{H}]+$} & 2.51 & 117.066 & 117.0657 & 45.0339 & 86.0599 & 57.0569 & 117.0661 & 91.0542 \\
\hline N-Phenylurea & $\mathrm{C} 7 \mathrm{H} 8 \mathrm{~N} 2 \mathrm{O}$ & $64-10-8$ & {$[\mathrm{M}+\mathrm{H}]+$} & 3.84 & 137.071 & 137.0708 & 77.0389 & 104.0496 & 51.0228 & 119.0604 & 137.0714 \\
\hline Phthalic acid & $\mathrm{C} 8 \mathrm{H} 6 \mathrm{O} 4$ & $88-99-3$ & {$[\mathrm{M}-\mathrm{H}]-$} & 3.67 & 165.019 & 165.0193 & 77.0394 & 121.0296 & 96.9602 & 164.8977 & 147.8929 \\
\hline
\end{tabular}


Table S- 5 continued.

\begin{tabular}{|c|c|c|c|c|c|c|c|c|c|c|c|}
\hline Phthalic anhydride & $\mathrm{C} 8 \mathrm{H} 4 \mathrm{O} 3$ & $85-44-9$ & {$[\mathrm{M}+\mathrm{H}]+$} & 15.98 & 149.023 & 149.0232 & 65.0388 & 39.0229 & $\begin{array}{c}121.029 \\
3\end{array}$ & 84.9602 & 149.0238 \\
\hline Phthalimide & $\mathrm{C} 8 \mathrm{H} 5 \mathrm{NO} 2$ & $85-41-6$ & {$[\mathrm{M}+\mathrm{H}]+$} & 5.43 & 148.039 & 148.0392 & 130.0336 & 102.0341 & 75.0235 & 120.0446 & 51.0239 \\
\hline Picolinafen & $\mathrm{C} 19 \mathrm{H} 12 \mathrm{~F} 4 \mathrm{~N} 2 \mathrm{O} 2$ & $\begin{array}{c}137641-05- \\
5\end{array}$ & {$[\mathrm{M}+\mathrm{H}]+$} & 17.02 & 377.091 & 148.0392 & 102.0339 & 30.0331 & 75.0237 & 148.0400 & - \\
\hline Prothioconazole & $\mathrm{C} 14 \mathrm{H} 15 \mathrm{Cl} 2 \mathrm{~N} 3 \mathrm{OS}$ & $\begin{array}{c}178928-70- \\
6\end{array}$ & {$[\mathrm{M}+\mathrm{H}]+$} & 14.74 & 344.039 & 344.0387 & 98.9843 & 125.0152 & $\begin{array}{c}189.047 \\
1\end{array}$ & 326.0312 & - \\
\hline Py-ethylamine & C8H8CIF3N2 & - & {$[\mathrm{M}+\mathrm{H}]+$} & 4.51 & 225.04 & 225.0400 & 196.0136 & 208.0142 & $\begin{array}{c}172.037 \\
4\end{array}$ & 160.0371 & 30.0339 \\
\hline Salicylic acid & $\mathrm{C} 7 \mathrm{H} 6 \mathrm{O} 3$ & $69-72-7$ & {$[\mathrm{M}-\mathrm{H}]-$} & 6.29 & 137.024 & 137.0243 & 93.0348 & 65.0399 & - & - & - \\
\hline Spiroxamine & $\mathrm{C} 18 \mathrm{H} 35 \mathrm{NO} 2$ & $\begin{array}{c}118134-30- \\
8\end{array}$ & {$[\mathrm{M}+\mathrm{H}]+$} & 12.00 & 298.274 & 298.2739 & 144.1379 & 100.1120 & $\begin{array}{c}126.127 \\
8\end{array}$ & - & - \\
\hline Tebuconazole & $\mathrm{C} 16 \mathrm{H} 22 \mathrm{CIN} 3 \mathrm{O}$ & $\begin{array}{c}107534-96- \\
3\end{array}$ & {$[\mathrm{M}+\mathrm{H}]+$} & 14.94 & 308.152 & 308.1524 & 70.0397 & 125.0155 & $\begin{array}{c}151.031 \\
3\end{array}$ & 165.0467 & - \\
\hline Tetraethyleneglycoldimethylether/ Tetraglyme & $\mathrm{C} 10 \mathrm{H} 22 \mathrm{O} 5$ & $143-24-8$ & {$[\mathrm{M}+\mathrm{H}]+$} & 6.82 & 223.154 & 223.1540 & 59.0489 & 103.0755 & $\begin{array}{c}207.033 \\
1\end{array}$ & 191.0017 & - \\
\hline Thiacloprid & $\mathrm{C} 10 \mathrm{H} 9 \mathrm{CIN} 4 \mathrm{~S}$ & $\begin{array}{c}\text { 111988-49- } \\
9\end{array}$ & {$[\mathrm{M}+\mathrm{H}]+$} & 8.63 & 253.031 & 253.0305 & 126.0108 & 90.0340 & - & - & - \\
\hline Thiourea & $\mathrm{CH} 4 \mathrm{~N} 2 \mathrm{~S}$ & $62-56-6$ & {$[M+H]+$} & 0.71 & 77.017 & 77.0168 & 59.9901 & 43.0291 & 56.9638 & - & - \\
\hline Triethylamine & $\mathrm{C} 6 \mathrm{H} 15 \mathrm{~N}$ & $121-44-8$ & {$[\mathrm{M}+\mathrm{H}]+$} & 1.88 & 102.128 & 102.1275 & 58.0649 & 102.1280 & 74.0968 & 30.0339 & 44.0496 \\
\hline Triethyleneglycoldimethylether/ Triglyme & $\mathrm{C} 8 \mathrm{H} 18 \mathrm{O} 4$ & $112-49-2$ & {$[\mathrm{M}+\mathrm{H}]+$} & 5.80 & 179.128 & 179.1277 & 59.0493 & 103.0759 & 977.0399 & 133.0658 & - \\
\hline Trimethylcarboxylic acid & $\mathrm{C} 12 \mathrm{H} 13 \mathrm{NO} 2$ & - & {$[\mathrm{M}+\mathrm{H}]+$} & 8.80 & 204.102 & 204.1015 & 188.0706 & 174.0552 & 244.0810 & 160.1124 & 143.0728 \\
\hline
\end{tabular}




\section{RESULTS OF TRAINING DATA}

\subsection{Overview of Detection of ISTD in Training Data Set}

Table S-6 - List of labelled internal standards screened in positive and negative ionisation mode, detected ion/adduct, retention time, sample matches $(n=29)$ and trend.

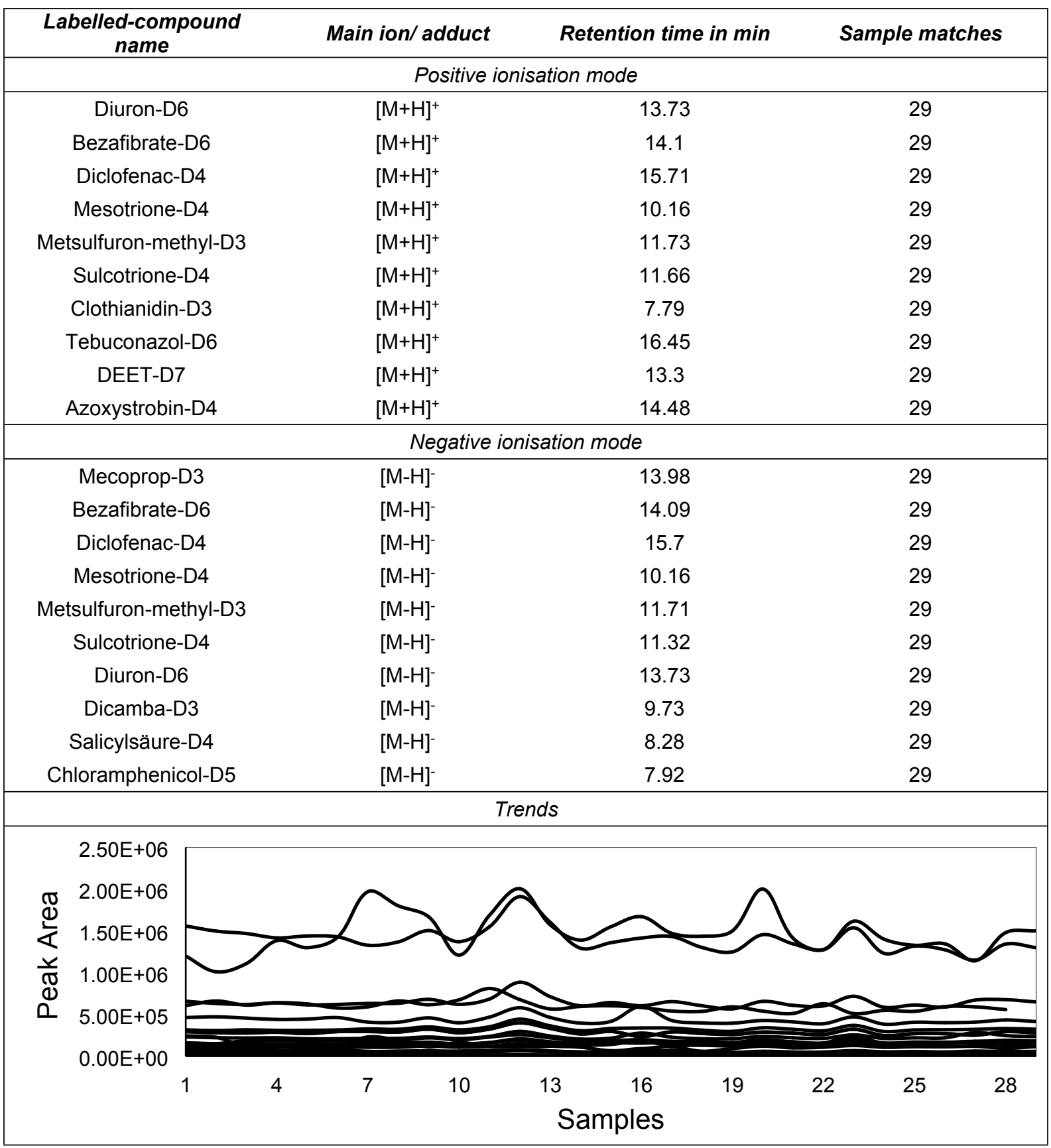




\subsection{Column and row-wise PCA}

Figure S-1 (see SI 3.2) shows the scatter plot of Q-statistic versus D-statistic for exploration of features and identification of highly leveraged points (for example features 95, 40, 78 and 119 which exceed D critical limit and features 132, 84 and 47 which exceed Q-critical limit).

Computing Tscore for time points ( Tscore $_{t}$ ) by row-wise PCA is the first evaluation step to show these time points which need further attention. Analysing target data, the time points (samples) 1,3 and 20 of synthetic samples present higher scores which were justified considering the concentration of spiked compounds and their trend type. Also, an increasing trend is evident from time point 21 onward (cf. Figure S-2). In the following section, GPCA processing shows while more regular shifts in Tscore $_{t}$ are due to increasing/decreasing profiles, the sudden rise in time point 20 is mainly attributed to two GPC1 and GPC 8 (non-relevant compounds).

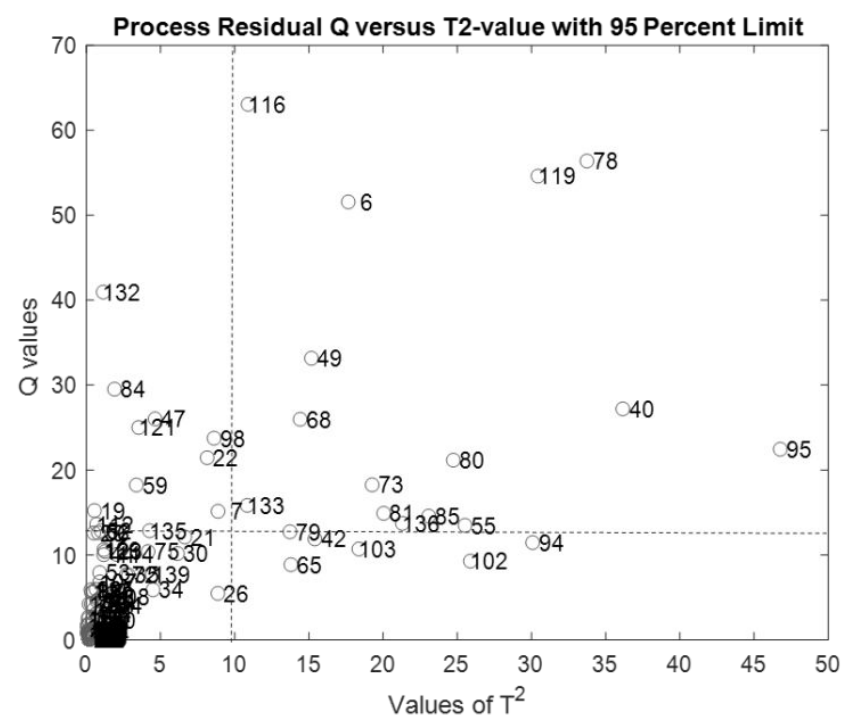

Figure S- 1 - Scatter plot of Q-values vs Hotelling's T2 values obtained by PCA of column-wise validation data.

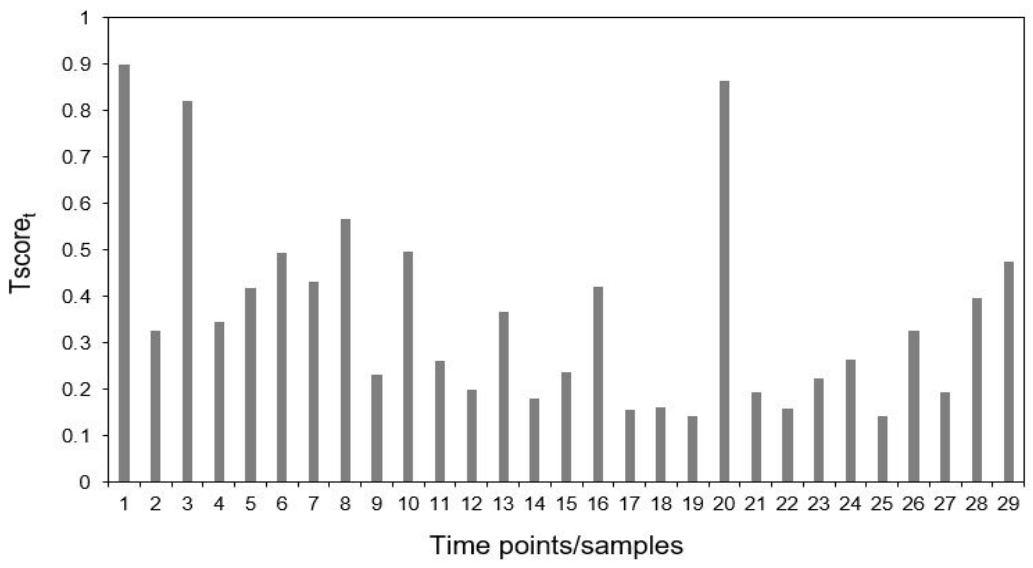

Figure S- 2 - Sscore $_{t}$ plot obtained by row-wise PCA processing of validation data. 
Table S- 7 - Detailed result of the 30 prioritised features (feature index, $\mathrm{m} / \mathrm{z}$ and retention time) of column-wise PCA (the result of 13PCs) in combination with Tscore $_{f}$ index. "Both trends" means first decreasing in intensity followed by increasing intensity changes over time. The spiked target analytes recovered are listed. Features determined as unknowns did not fit spiked target compounds.

\begin{tabular}{|c|c|c|c|c|c|}
\hline Index & $m / z$ & Retention time in min & Tscore & Trend & Spiked target compounds \\
\hline 95 & 362.0307 & 15.98 & 5.1984 & both trends & Prothioconazole \\
\hline 78 & 304.2608 & 20.63 & 4.1562 & peaks-increasing & Unknown \\
\hline 40 & 201.0691 & 9.23 & 3.8173 & fluctuating & N,N-Dimethyl-N'-phenylsulfamid/ DMSA \\
\hline 94 & 360.0334 & 15.98 & 3.2173 & both trends & Prothioconazole \\
\hline 102 & 404.1238 & 14.54 & 2.4692 & increasing & Azoxystrobin \\
\hline 55 & 237.1016 & 12.55 & 2.4297 & decreasing & Carbamazepine \\
\hline 80 & 310.1498 & 16.52 & 2.2700 & fluctuating & Tebuconazole \\
\hline 85 & 314.0638 & 15.36 & 2.2260 & decreasing & Cyproconazol \\
\hline 6 & 137.0343 & 5.01 & 2.1450 & fluctuating & $\mathrm{ABC} 700$ \\
\hline 73 & 292.1207 & 15.39 & 2.0146 & decreasing & Cyproconazole \\
\hline 103 & 426.1063 & 14.55 & 1.8719 & increasing & Azoxystrobin \\
\hline 81 & 312.0663 & 15.36 & 1.8696 & decreasing & Cyproconazol \\
\hline 119 & 223.9998 & 7.21 & 1.6900 & fluctuation & Clothianidin \\
\hline 68 & 282.2786 & 20.63 & 1.6878 & peaks-increasing & Unknown \\
\hline 42 & 201.1093 & 7.47 & 1.6399 & decreasing & Triethyleneglycoldimethylether/ Triglyme \\
\hline 136 & 358.0187 & 15.96 & 1.6147 & both trends & Prothioconazole \\
\hline 49 & 214.1202 & 13.41 & 1.5438 & fluctuating & N,N-Diethyl-3-methylbenzamide/ DEET \\
\hline 65 & 259.0841 & 12.56 & 1.4375 & decreasing & Carbamazepine \\
\hline 79 & 308.1524 & 16.51 & 1.1794 & fluctuating & Tebuconazole \\
\hline 22 & 177.0464 & 5.11 & 1.0005 & fluctuating & $\mathrm{ABC} 700$ \\
\hline 98 & 372.0983 & 14.55 & 0.9687 & increasing & Azoxystrobin \\
\hline 26 & 179.127 & 7.49 & 0.9497 & decreasing & Triethyleneglycoldimethylether/ Triglyme \\
\hline 7 & 137.0343 & 5.12 & 0.9299 & fluctuating & ABC 700 \\
\hline 21 & 170.041 & 6.83 & 0.8850 & peaks & 2-(Trifluoromethyl)benzamide \\
\hline 133 & 342.0239 & 16.33 & 0.8412 & both trends & Prothioconazole \\
\hline 30 & 190.0472 & 6.85 & 0.7922 & peaks & 2-(Trifluoromethyl)benzamide \\
\hline 116 & 175.0324 & 5.06 & 0.7726 & fluctuating & 2-(Trifluormethyl)benzenesulfonamide/ TBSA \\
\hline 34 & 194.0962 & 12.55 & 0.5401 & decreasing & Carbamazepine \\
\hline 47 & 210.1099 & 7.46 & 0.5236 & peaks-decreasing & Triethyleneglycoldimethylether/ Triglyme \\
\hline 75 & 294.1181 & 15.38 & 0.5147 & decreasing & Cyproconazol \\
\hline
\end{tabular}




\subsection{Results of GPCA}

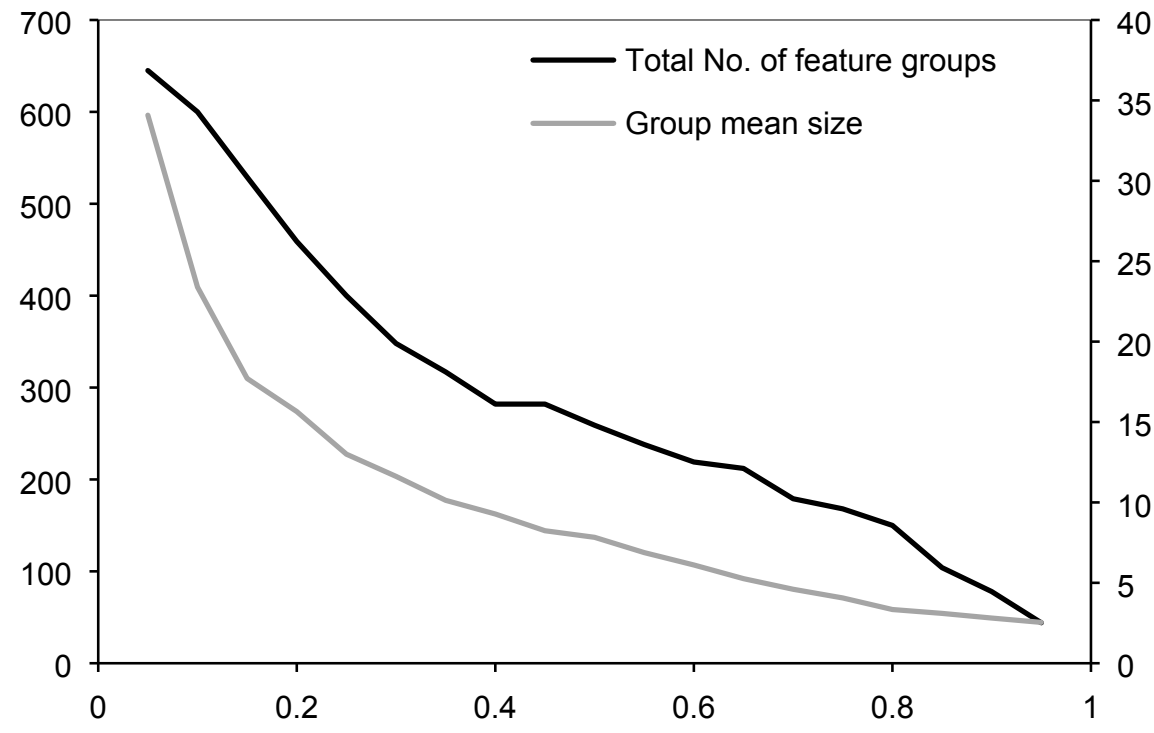

Figure S- 3 - Total number of feature groups and mean number of features per group as a function of selected threshold $y$ from the correlation map. The smaller the mean size of the group, the greater the sparsity. Nevertheless, by increasing the threshold value, some trends will be missed. So, initial experience about the association patterns of mass fragments is significant to select threshold value. 

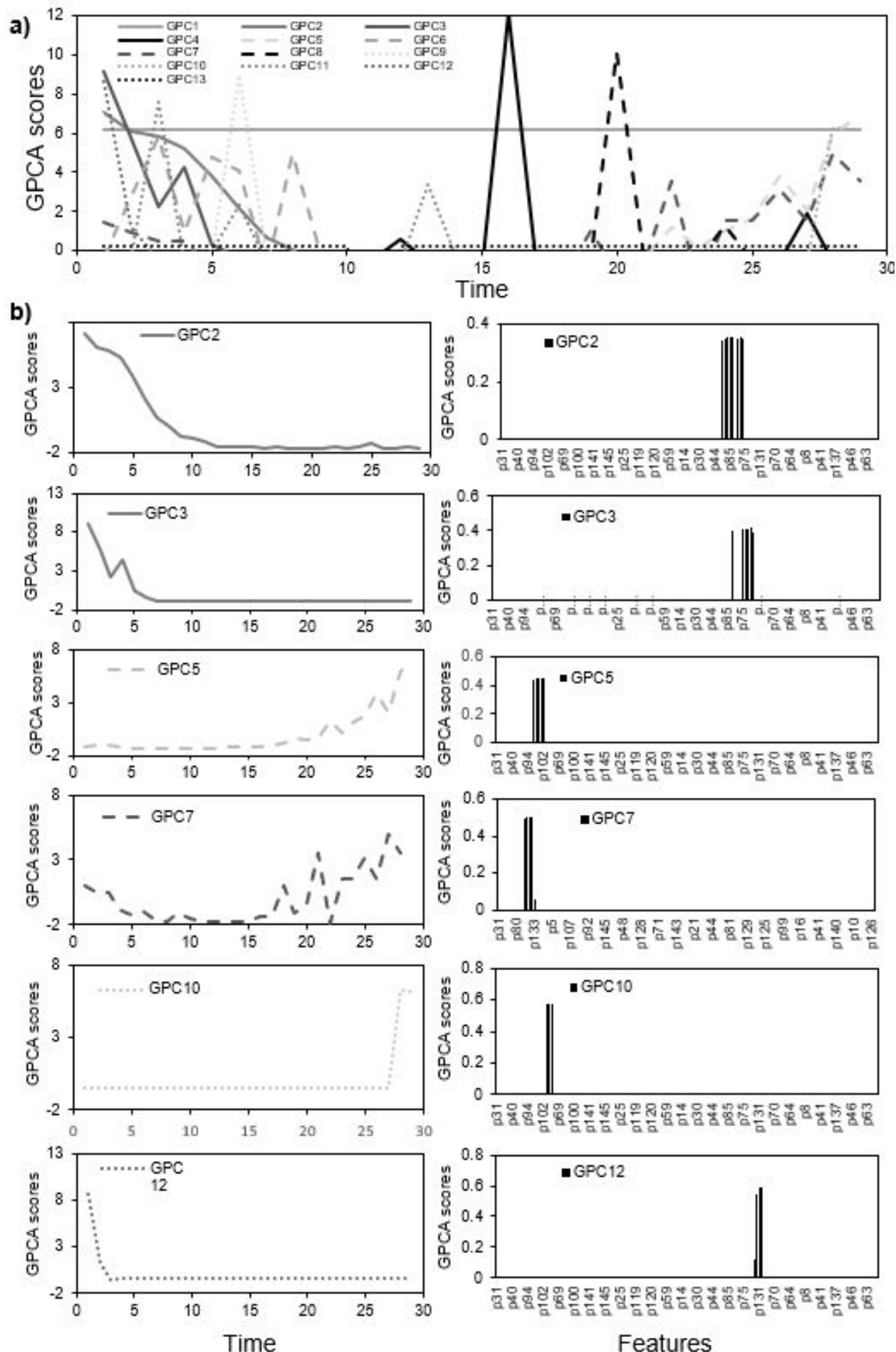

Figure S- 4 - Score vectors of first 13 components from the GPCA model for the validation (target) dataset (a). Scores and loadings for the six prioritised decreasing/increasing trends using groups obtained from GPCA (b). 


\section{Supporting Information}

Table S- 8- The effect of selecting different $\gamma$ value on recovering different groups of correlated features for TrOC $s^{1}$ by GPCA modelling of validation (target) data.

\begin{tabular}{|c|c|c|c|c|c|c|c|c|c|c|c|c|c|c|}
\hline \multirow[t]{2}{*}{ Trends } & \multicolumn{2}{|r|}{$\mathrm{DT}^{2}$} & \multicolumn{2}{|r|}{$\mathrm{BT}^{3}-\mathrm{IT}$} & \multicolumn{2}{|r|}{ DT } & \multicolumn{2}{|r|}{ DT } & \multicolumn{2}{|r|}{ BT-IT } & \multicolumn{2}{|r|}{$1 \mathrm{TT}^{4}$} & \multicolumn{2}{|r|}{ DT } \\
\hline & GPC2 & 3TrOCs & GPC4 & 2TrOCs & GPC5 & 2TrOCs & GPC7 & 2TrOCs & GPC9 & 2TrOCs & GPC10 & 1TrOCs & GPC12 & 1TrOCs \\
\hline \multirow{11}{*}{$\begin{array}{l}\stackrel{0}{0} \\
\stackrel{1}{>}\end{array}$} & 65 & (1)Carbamazepin & 139 & (4)Prothioconazole & 12 & Carbamazepin & 129 & Cyproconazole & 96 & Prothioconazole & p98 & (7)Azoxystrobin & 131 & Cyproconazole \\
\hline & 42 & (2)Triglyme & 136 & Prothioconazole & 28 & Carbamazepin & 76 & Cyproconazole & 90 & Prothioconazole & p103 & Azoxystrobin & 11 & Cyproconazole \\
\hline & 55 & Carbamazepin & 94 & Prothioconazole & 120 & Cyproconazole & 130 & Cyproconazole & 5 & (6)Diglyme & p102 & Azoxystrobin & 22 & Cyproconazole \\
\hline & 85 & (3)Cyproconazole & 95 & Prothioconazole & 54 & Cyproconazole & 106 & Carbamazepin & & & & & & \\
\hline & 81 & Cyproconazole & 133 & (5)Lactofen & 47 & Carbamazepin & & & & & & & & \\
\hline & 35 & Triglyme & 135 & Lactofen & & & & & & & & & & \\
\hline & 34 & Carbamazepin & & & & & & & & & & & & \\
\hline & 26 & Triglyme & & & & & & & & & & & & \\
\hline & 73 & Cyproconazole & & & & & & & & & & & & \\
\hline & 75 & Cyproconazole & & & & & & & & & & & & \\
\hline & 72 & Cyproconazole & & & & & & & & & & & & \\
\hline $\begin{array}{l}\text { Recall } \\
\text { Rate }^{5}\end{array}$ & GPC2 & $\begin{array}{l}(1) 43 \%,(2) 100 \% \\
\text { (3) } 36 \%\end{array}$ & GPC4 & (4)67\%, (5)100\% & GPC5 & (1)43\% (3)14\% & GPC7 & $\begin{array}{l}\text { (3)21\%, } \\
(100 \%) 14 \%\end{array}$ & GPC9 & $\begin{array}{l}\text { (4)33\% (100\%) } \\
\text { (6)100\% }\end{array}$ & GPC10 & (7)100\% & GPC12 & (3)21\% (92\%) \\
\hline \multirow[t]{2}{*}{ Trends } & DT & & DT & & IT & & DT & & BT & & IT-BT & & DT & \\
\hline & GPC2 & 3TrOCs & GPC3 & 2TrOCs & GPC5 & 2TrOCs & GPC6 & 2TrOCs & GPC7 & 1TrOCs & GPC10 & 2TrOCs & GPC12 & 1TrOCs \\
\hline \multirow{8}{*}{$\stackrel{\hat{0}}{\stackrel{0}{>}}$} & 26 & Triglyme & 35 & Triglyme & 98 & Azoxystrobin & 12 & Carbamazepin & 94 & Prothioconazole & 5 & Diglyme & 11 & Cyproconazole \\
\hline & 34 & Carbamazepin & 72 & Cyproconazole & 102 & Azoxystrobin & 28 & Carbamazepin & 95 & Prothioconazole & 90 & Prothioconazole & 106 & Cyproconazole \\
\hline & 42 & Triglyme & 75 & Cyproconazole & 103 & Azoxystrobin & 47 & Carbamazepin & 136 & Prothioconazole & 96 & Prothioconazole & 130 & Cyproconazole \\
\hline & 55 & Carbamazepin & 76 & Cyproconazole & 133 & Lactofen & 54 & Cyproconazole & 139 & Prothioconazole & & & 131 & Cyproconazole \\
\hline & 65 & Carbamazepin & 129 & Cyproconazole & 135 & Lactofen & 120 & Cyproconazole & & & & & & \\
\hline & 73 & Cyproconazole & 130 & Cyproconazole & & & & & & & & & & \\
\hline & 81 & Cyproconazole & & & & & & & & & & & & \\
\hline & 85 & Cyproconazole & & & & & & & & & & & & \\
\hline $\begin{array}{l}\text { Recall } \\
\text { Rate }\end{array}$ & GPC2 & $\begin{array}{l}\text { (1) } 43 \%,(2) 67 \% \\
\text { (3) } 21 \%\end{array}$ & GPC3 & $\begin{array}{l}\text { (2)33\% (100\%) } \\
(3) 36 \%\end{array}$ & GPC5 & $\begin{array}{l}7) \mathbf{1 0 0 \%} \\
(5) \mathbf{1 0 0} \%\end{array}$ & GPC6 & $\begin{array}{l}\text { (1)43\% (86\%) } \\
\text { (3)14\% }\end{array}$ & GPC7 & (4)67\% & GPC10 & $\begin{array}{l}6) 100 \% \\
(4) 33 \%(100 \%)\end{array}$ & GPC12 & (3)29\% (100\%) \\
\hline \multirow[t]{2}{*}{ Trends } & DT & & IT & & BT & & DT & & DT & & BT-IT & & DT & \\
\hline & GPC2 & 3TrOCs & GPC4 & 2TrOCs & GPC6 & 1TrOCs & GPC7 & 2TrOCs & GPC9 & 2TrOCs & GPC10 & 2TrOCs & GPC12 & 1TrOCs \\
\hline \multirow{5}{*}{$\stackrel{\infty}{\infty}$} & p65 & Carbamazepin & p133 & Lactofen & p139 & Prothioconazole & p12 & Carbamazepin & p35 & Triglyme & p96 & Prothioconazole & p129 & Cyproconazole \\
\hline & $\mathrm{p} 42$ & Triglyme & p135 & Lactofen & p136 & Prothioconazole & p28 & Carbamazepin & p75 & Cyproconazole & p90 & Prothioconazole & p76 & Cyproconazole \\
\hline & p55 & Carbamazepin & p98 & Azoxystrobin & p94 & Prothioconazole & p120 & Cyproconazole & p72 & Cyproconazole & p5 & Diglyme & p130 & Cyproconazole \\
\hline & p85 & Cyproconazole & p103 & Azoxystrobin & p95 & Prothioconazole & p54 & Cyproconazole & & & & & & \\
\hline & $\mathrm{p} 81$ & Cyproconazole & p102 & Azoxystrobin & & & & & & & & & & \\
\hline & $\frac{\mathrm{p} 26}{\mathrm{GPC} 2}$ & $\begin{array}{l}\text { Triglyme } \\
\text { (1)29\%, (2)67\% }\end{array}$ & GPC4 & (5)100\% & GPC5 & (4) $67 \%$ & GPC6 & (1) $29 \%(58 \%)$ & GPC7 & (2)33\% (100\%) & GPC10 & $(4) 33 \%(\mathbf{1 0 0 \%})$ & GPC12 & (3)21\% (63\%) \\
\hline $\begin{array}{l}\text { Rate } \\
\text { Rate }\end{array}$ & & (3) $14 \%$ & & (7) $100 \%$ & & & & (3) $14 \%$ & & (3) $14 \%$ & & 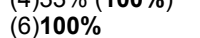 & & \\
\hline \multirow[t]{2}{*}{ Trends } & DT & & DT & & IT & & BT-IT & & DT & & & & & \\
\hline & GPC2 & 3TrOCs & GPC3 & 3TrOCs & GPC6 & 1TrOCs & GPC7 & 2TrOCs & GPC12 & 1TrOCs & & & & \\
\hline \multirow{6}{*}{$\begin{array}{l}\stackrel{9}{0} \\
\stackrel{1}{\longrightarrow} \\
\rightarrow\end{array}$} & p35 & Triglyme & p42 & Triglyme & p98 & Azoxystrobin & p96 & Prothioconazole & p129 & Cyproconazole & & & & \\
\hline & p34 & Carbamazepin & p55 & Carbamazepin & p103 & Azoxystrobin & p90 & Prothioconazole & p76 & Cyproconazole & & & & \\
\hline & p26 & Triglyme & p85 & Cyproconazole & p102 & Azoxystrobin & p5 & Diglyme & & & & & & \\
\hline & $\mathrm{p} 73$ & Cyproconazole & p81 & Cyproconazole & & & & & & & & & & \\
\hline & p75 & Cyproconazole & & & & & & & & & & & & \\
\hline & p72 & Cyproconazole & & & & & & & & & & & & \\
\hline $\begin{array}{l}\text { Recall } \\
\text { Rate }\end{array}$ & GPC2 & $\begin{array}{l}\text { (1) } 14 \%,(2) 67 \% \\
\text { (3) } 21 \%\end{array}$ & GPC3 & $\begin{array}{l}\text { (2)33\% (100\%) } \\
\text { (1)14\% (28\%) } \\
\text { (3)14\% }\end{array}$ & GPC6 & (7)100\% & GPC6 & $\begin{array}{l}(4) 33 \% \\
\text { (6)100\% }\end{array}$ & GPC7 & (3)14\% (49\%) & (5)N.D. & & & \\
\hline
\end{tabular}

1TrOCs stands for trace organic compounds.

${ }_{2,3,4} \mathrm{DT}, \mathrm{BT}$ and IT stand for decreasing trends, both trends and increasing trends, respectively.

${ }^{5}$ Recall rate or true positive rate was calculated as: True number of detected trends/total number of trends.

${ }^{6}$ The values shown in bold, present the highest percentage of recovered features for each TrOC. 


\section{Supporting Information}

Table S- 9 - Groups of recorded features by GPCA modelling of validation (target) data $(\gamma=0.7)$.

\begin{tabular}{|c|c|c|c|c|c|}
\hline Variance \% & $\begin{array}{c}\text { Cumulative } \\
\text { variance }\end{array}$ & Trend & GPC & Feature indices & Target analyte \\
\hline 27.97 & 27.97 & Monotone line & GPC1 & $\begin{array}{l}2,8,9,10,13,16,17,18,20,23,24,27,33,39,41,43,45,46,57,62,63,64, \\
86,88,89,91,93,99,105,111,115,118,124,126,127,134,137,138,140,144\end{array}$ & Unknown \\
\hline 5.328 & 33.30 & Decreasing trend & GPC2 & $26,34,42,55,65,73,81,85$ & Carbamazepine, Triglyme, Cyproconazole \\
\hline 3.994 & 37.29 & Decreasing trend & GPC3 & $35,72,75,76,129,130$ & Triglyme, Cyproconazole \\
\hline 3.970 & 41.26 & Single peak & GPC4 & $92,113,141,142,148,149$ & Unknown \\
\hline 3.341 & 44.60 & Increasing trend & GPC5 & $98,102,103,133,135$ & Azoxystrobin, Lactofen \\
\hline 3.277 & 47.88 & Decreasing Trend & GPC6 & $12,28,47,54,120$ & Carbamazepine, Cyproconazole \\
\hline 2.714 & 50.60 & Both trends & GPC7 & $94,95,133,136,139$ & Prothioconazole \\
\hline 2.716 & 53.31 & Single peak & GPC8 & $68,69,78,108$ & Unknown \\
\hline 2.098 & 55.41 & Single peak & GPC9 & $3,14,97$ & Thiacloprid, unknown \\
\hline 2.068 & 57.48 & Increasing trend & GPC10 & $5,90,96$ & Diglyme, Prothioconazole \\
\hline 2.052 & 59.53 & Peaks & GPC11 & $52,56,59$ & Tetraglyme \\
\hline 1.982 & 61.51 & Decreasing Trend & GPC12 & $11,106,130,131$ & Cyproconazole \\
\hline 1.399 & 62.91 & Single peak & GPC13 & 19,29 & 2-(Trifluoromethyl)benzamide \\
\hline
\end{tabular}




\section{RSULTS OF WASTEWATER SAMPLES}

\subsection{Analysis of QC Samples}

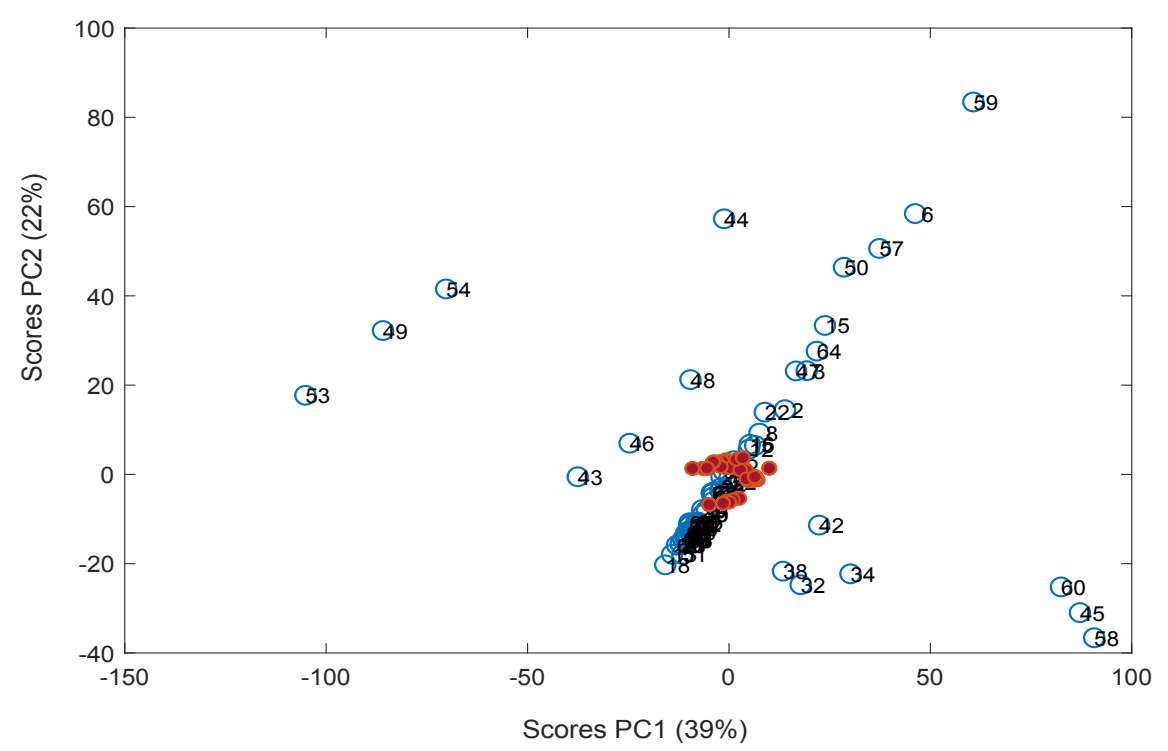

Figure S- 5-2D scores plot obtained by row-wise PCA (which displays the observations, here the samples) of QC samples (red circles) and wastewater samples (blue circles).

\subsection{Pre-Filtering of Raw Data Matrix}

For pre-filtering of wastewater samples data matrices, implementation of two univariate statistics were first evaluated on target data: the Spearman rank's correlation and the Mann-Kendall test (MK, which was initially used for climate factors analysis ${ }^{9}$ and rainfall trend analysis ${ }^{10}$ or in the machine learning research ${ }^{11}$ ). At first, each of 149 target features (belong to 19 spiked target pollutants) was manually assigned to the increasing/decreasing trends or other trends. Then, the time trend intensity of each feature has been compared with statistical results, individually (a p-value smaller than the significance level of 0.05 was considered as a trend with significantly change with time) ${ }^{11} .75 \%$ of determined time trends determined by MK confirmed the spiked time patterns, which results in $25 \%$ false-negative MK determined time trends. Features which were not detected by MK had monotonic trends, showed both increasing and decreasing trend or having significant fluctuation while falling/increasing (cf. Figure S-6). The false-negative results comprised, among others, the prioritised features by Tscore and GPCA. However, MK Test represents both increasing and decreasing trend in the intensity of features over time, although not much significant, as also determined by Modal et al. ${ }^{10}$. In comparison, the true and false detected trends using Spearman's rank correlation test were $94 \%$ and $6 \%$, respectively. In other studies, the statistical performance from both univariate statistical tests, were more consistent with each other ${ }^{12-14}$. 
In addition, six target compounds with increasing/decreasing trend would produce 27 relevant features, which 24 of them (recall rate of $88 \%$ ) was selected using only p-value parameter using the latter method. On the other hand, inspecting the recovered decreasing/increasing GPCs of the whole target data showed that all false non-significant trends ( 3 out of 27 features) did have a correlation coefficient of around 0.32. Therefore, with the aim of not losing any relevant feature, a threshold of $|\rho| \geq 0.3$ ( $88 \%$ p-values $<0.05)$, with a false-negative rate of zero or recall rate of $100 \%$ and specificity of $77 \%$, was selected as the optimum value for pre-filtering the initial raw data matrices. In Table S-10, confusion matrices obtained through setting different correlation cut-off values of $0.2,0.3$ and 0.4 have been shown. So, based on a preliminary evaluation of the data, these three cut-off values of $0.2,0.3$ and 0.4 were selected and the features that showed lower or equal/higher than cut-off values were discriminated, and recall rate or true positive rate and specificity values were calculated based on the confusion matrices. As shown in Table S-10, by increasing the threshold value from 0.3 to 0.4 , the recall rate was reduced to $89 \%$, so the higher this threshold, the more relevant features are lost. As the aim was not losing any relevant feature, a threshold of $\rho=0.3$, with a false-negative rate of zero or recall rate of $100 \%$ and specificity of $77 \%$, was selected as the optimum value for pre-filtering of the initial raw data matrices.

Figure S-7 shows a 2D score plot of features in PC2-PC1 space. Here, for example, features 1315 and 1410, 230 and 1198, 1870 and 1978 and 2223 located at different parts of the score plot, show a fluctuating pattern, peak pattern and an increasing trend $(\rho=0.68)$, respectively. The results of applying the PCA on the pre-filtered and pre-processed data matrices, as 2D score and loading plots for the first two principal components, are shown in Figure S-8 in both ionisation modes.

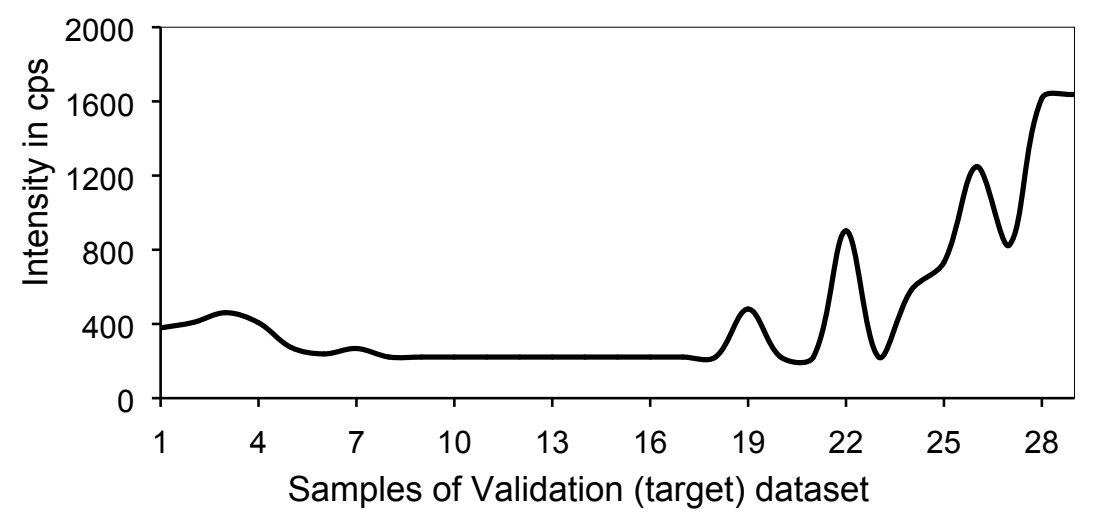

Figure S- 6 - Example of MK failed monotonic time trend test. Patterns with fluctuation in increasing/decreasing part of the trend cause failure in the MK test. The example shows the feature with ID 133 in positive ionisation mode. The $p$-value is 0.069 , indicating no trend, according to $M K(p>0.05)$. 
Table S- 10 - Confusion matrices according to different threshold values for selecting the optimum Spearman's rank correlation cut-off, based on validation (target) data.

\begin{tabular}{|c|c|c|c|c|c|c|c|}
\hline \multirow[t]{2}{*}{ Target Data } & \multirow[t]{2}{*}{ A priori } & \multicolumn{2}{|c|}{$|\rho|=0.2$} & \multicolumn{2}{|c|}{$|\rho|=0.3$} & \multicolumn{2}{|c|}{$|\rho|=0.4$} \\
\hline & & Up/down & Other trends & Up/down & Other trends & Up/down & Other trends \\
\hline Up/down trends & 27 & 27 & 0 & 27 & 0 & 24 & 3 \\
\hline Other trends & 122 & 46 & 76 & 28 & 94 & 12 & 110 \\
\hline Total & 149 & & $\begin{array}{l}=100 \% \\
*=62 \%\end{array}$ & & $R R=100 \%$ & & $\begin{array}{l}=89 \% \\
=90 \%\end{array}$ \\
\hline
\end{tabular}

" $R R$, stands for recall rate, $R R=T P / T P+F N$, which TP is true positive and FN is false negative.

"*P, stands for specificity, $\mathrm{SP}=\mathrm{TN} / \mathrm{TN}+\mathrm{FP}$, which TN is true negative and FP is false positive. 


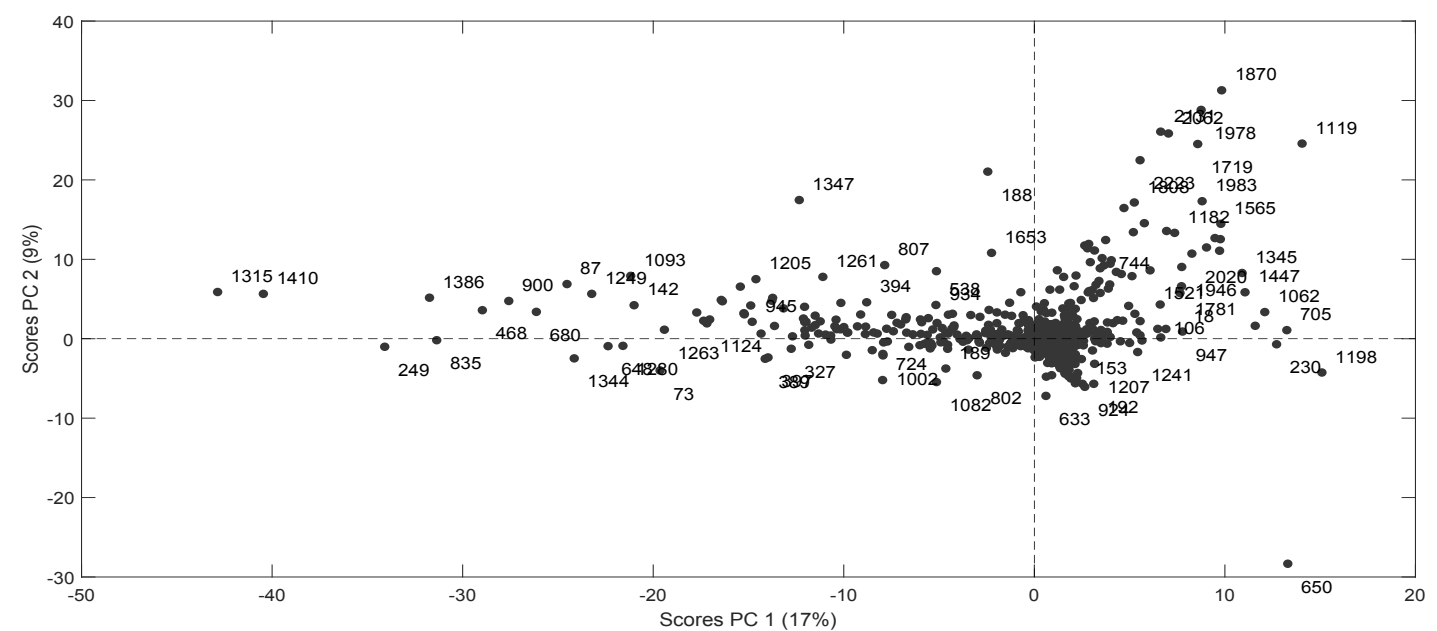

Figure S- 7-2D Score plot of the first two components derived from a PCA model based on LC-HRMS data matrix of influent WWTP sample in positive ionisation mode. Column-wise PCA model of pre-processed initial data matrix of positive ionisation mode, used 25 components to explain $80 \%$ of the total variance in the data.
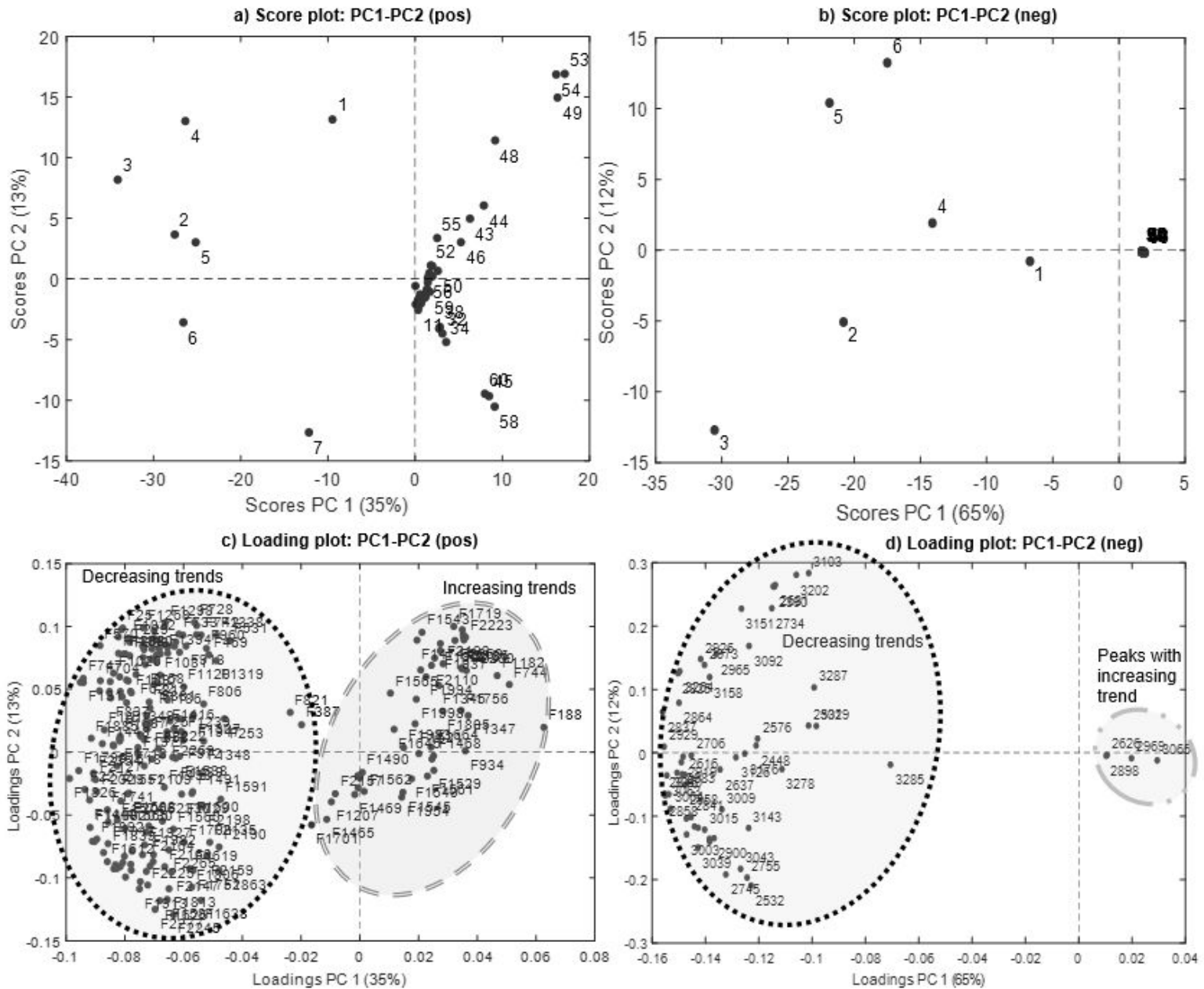

Figure S- $8-2 D$ score $(a \& b)$ and loading (c \&d) plots obtained by PCA modelling of pre-filtered WWTP influent data matrices containing increasing/decreasing intensity patterns for positive and negative ionisation mode, respectively. 


\subsection{Results of Prioritising Samples in Time Series by Row-wise PCA}

While a general overview regarding increasing and decreasing time trends in loading plots can be perfectly visualised, the definitive conclusion about the relationship between variables and time points (especially for increasing trends) is a hard task for complex data like industrial wastewaters. As an alternative visualisation of the problem, the evolution of Tscore $_{t}$ (validated for target data, see SI 3.3) indices with time points before and after the feature selection step is presented in Figure S-9. For the initial dataset and without filtering, the important time points can be detected of calculated Tscore $_{t}$ values. Here, the initial time points and time points $48-$ 59 have higher score values than the others. The result of Figure S- 9 focused the attention on the particular timeframe or even specific sampling times because the sudden presence of TrOCs in the environment could be a matter of concern. Therefore, data pre-filtering was performed to prioritise the time Tscore $_{t}$ points 2 to 7 and 48 to 60 .

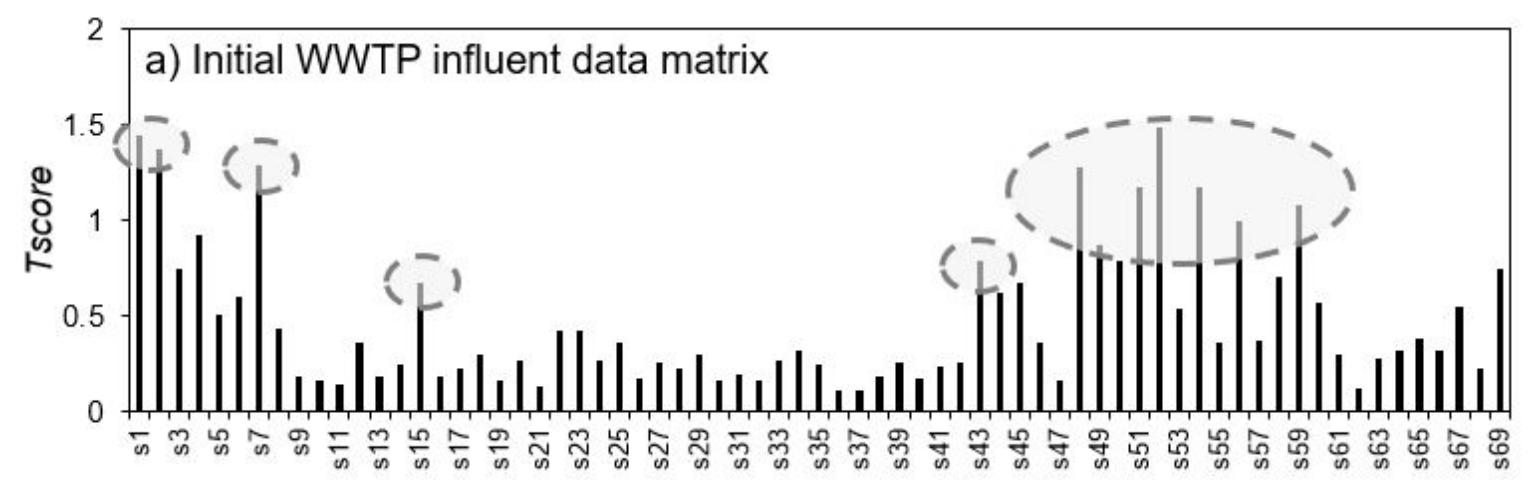

b) Prefiltered data matrix

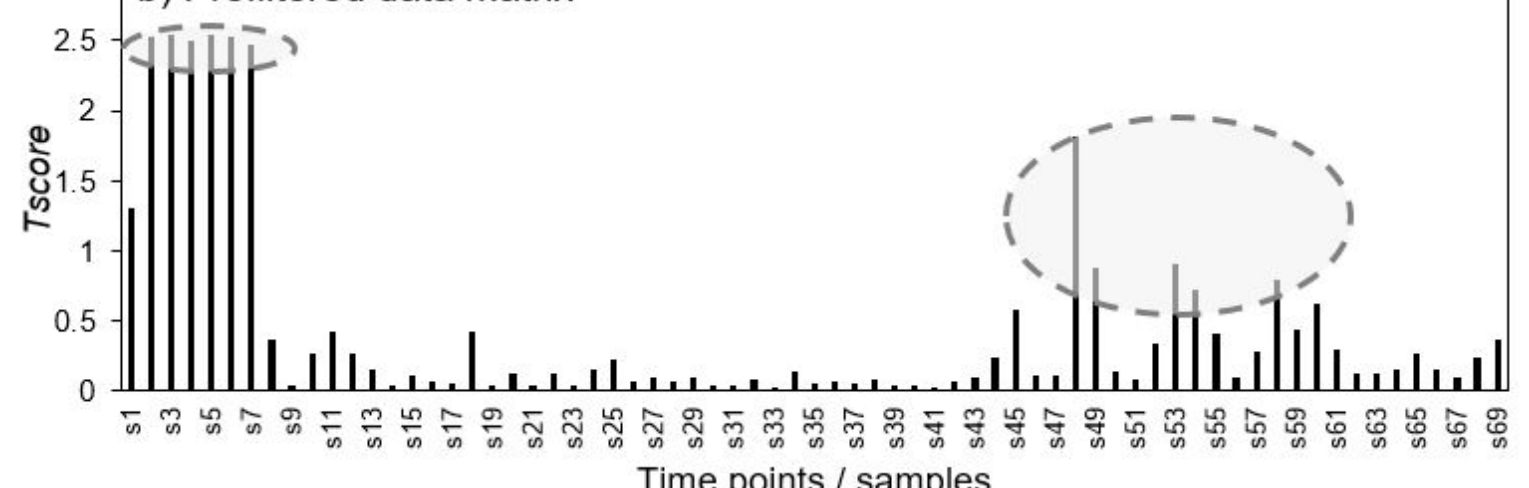

Time points / samples

Figure S- 9 - Tscore $_{t}$ values for PCA modelling of (a) initial WWTP influent data matrix (69*2380) in positive ionisation mode using 15 PCs with a total variance of $81.1 \%$ and (b) pre-filtered data matrix (69*234) based on 10 $P C s$ with a total variance of $87.3 \%$. The most relevant time points regarding high score values are indicated by dashed circles. 


\subsection{GPCA Results and Prioritised Features Responsible for Trend Phenomena using First Prioritisation Route}

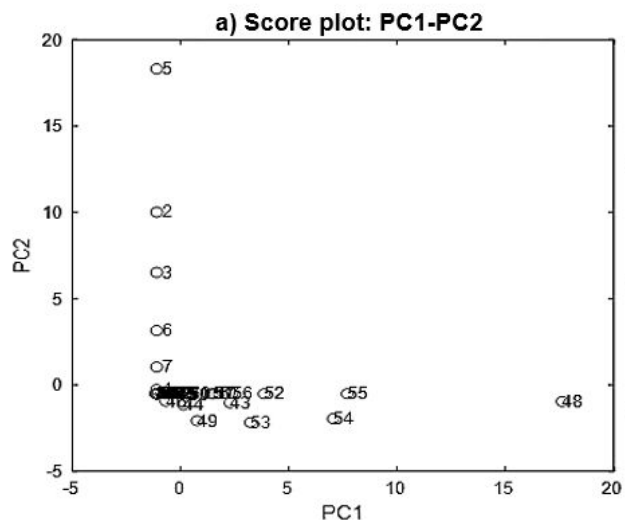

b) Line/bar plot: GPC1-GPC2

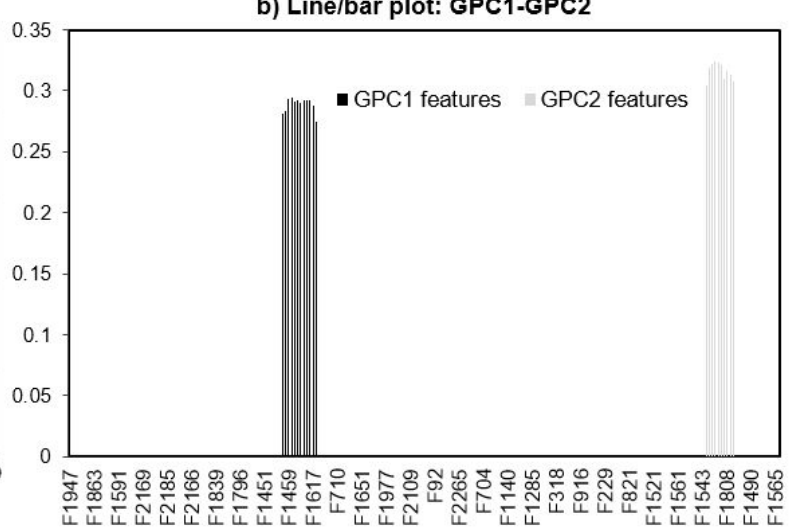

Figure S- $10-2 D$ score plot of first 2 PCs in GPCA of pre-filtered WWTP influent data in positive ionisation mode (a) and the corresponding loading (line/bar) plot (b).

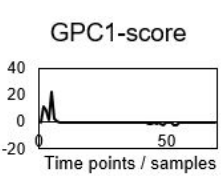

GPC7-score

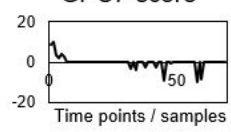

GPC13-score

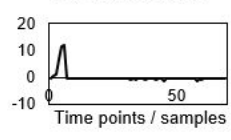

GPC19-score

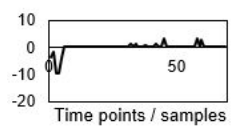
GPC25-score

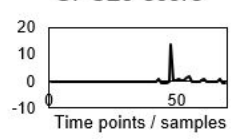

GPC2-score

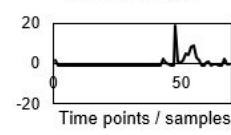

GPC8-score

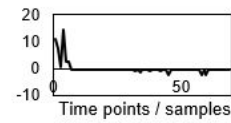

GPC14-score

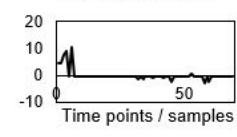

GPC20-score

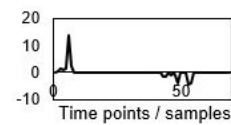

GPC3-score

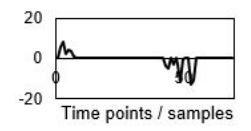

GPC9-score

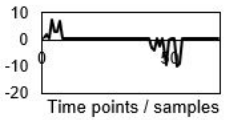

$$
\text { GPC15-score }
$$

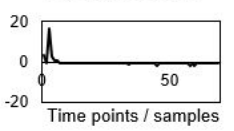

GPC21-score

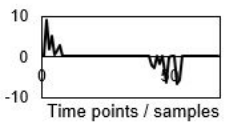

GPC4-score

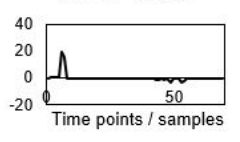

GPC10-score

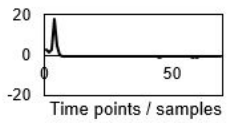

$$
\text { GPC16-score }
$$

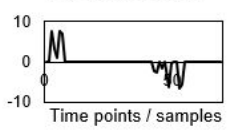

GPC22-score

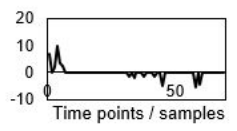

GPC5-score

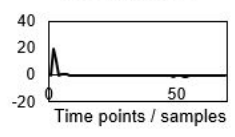

GPC11-score

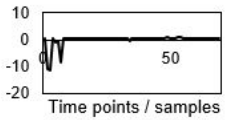

GPC17-score

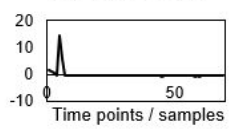

GPC23-score

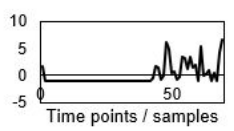

GPC6-score

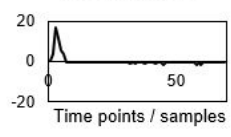

GPC12-score

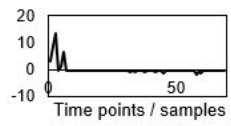

GPC18-score

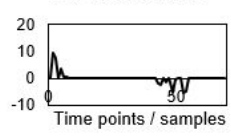

GPC24-score

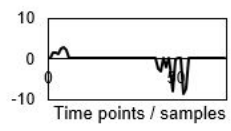

Figure S- 11 - Results of GPCA of positive ionisation mode. For example, the first score vector with the highest variance has a decreasing trend. The second GPC contains the most important features responsible for increasing trends, especially at time point/sample s48.

GPC1-score

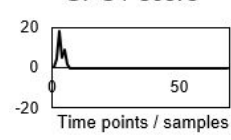

GPC7-score

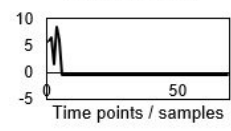

GPC2-score

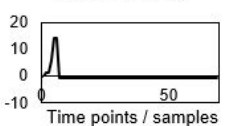

GPC8-score

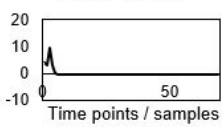

GPC3-score
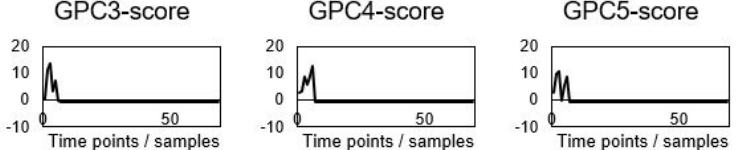

GPC6-score

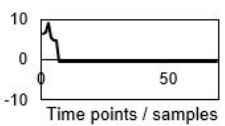

Figure S- 12 - Results of GPCA of negative ionisation mode. All GPCs belonged to decreasing trends. 
Table S- 11- Detailed list of features prioritised by GPCA. The features printed in thick are these ones following the relevant trend of continuously increasing/decreasing intensity. In total, 130 relevant features were prioritised.

\begin{tabular}{|c|c|c|c|c|c|c|c|c|c|c|c|c|}
\hline \multicolumn{13}{|c|}{ Prioritised features of WWTP influent samples in positive ionisation mode } \\
\hline GPC1 & F1457 & F1459 & F1618 & F1687 & F1778 & F1686 & F1713 & F1714 & F1617 & F1717 & F1538 & F924 \\
\hline GPC2 & F2062 & F1978 & F2131 & F1870 & $\mathbf{F} 2223$ & F1808 & F1937 & F1719 & F2031 & F2268 & - & - \\
\hline GPC3 & F1612 & F2121 & F2145 & F1935 & F2243 & F2166 & F1616 & F1747 & F1809 & F1581 & - & - \\
\hline GPC4 & $\mathrm{F} 2135$ & F2159 & F1729 & F1619 & F1600 & F2190 & F1752 & F1491 & F1591 & F2198 & - & - \\
\hline GPC5 & F2269 & F2307 & F2359 & F2109 & F153 & F725 & F1307 & F2222 & - & - & - & - \\
\hline GPC6 & F747 & F191 & F1253 & F1206 & F1197 & F1288 & F622 & F1380 & - & - & - & - \\
\hline GPC7 & F733 & F913 & F1232 & F742 & F916 & F1429 & F751 & F728 & - & - & - & - \\
\hline GPC8 & F1286 & F1214 & F959 & F960 & F1381 & F227 & F1529 & - & - & - & - & - \\
\hline GPC9 & F2225 & F2155 & F1716 & F2147 & F2185 & F2245 & F2169 & F2243 & - & - & - & - \\
\hline GPC10 & F1059 & F894 & F469 & F1285 & F1319 & F1947 & - & - & - & - & - & - \\
\hline GPC11 & F2359 & F2222 & $\mathrm{F} 2130$ & F1722 & F2061 & F1977 & F1873 & - & - & - & - & - \\
\hline GPC12 & F935 & F1240 & F1349 & F1097 & F192 & - & - & - & - & - & - & - \\
\hline GPC13 & F858 & F362 & F1041 & F710 & F837 & - & - & - & - & - & - & - \\
\hline GPC14 & F1020 & F398 & F1006 & F92 & F570 & F1032 & - & - & - & - & - & - \\
\hline GPC15 & F1120 & F806 & F1186 & F1140 & F1245 & F1206 & F1197 & - & - & - & - & - \\
\hline GPC16 & F1896 & F1917 & F1913 & F1488 & F1574 & - & - & - & - & - & - & - \\
\hline GPC17 & F939 & F1239 & F1348 & F1416 & $\mathrm{F} 1713$ & - & - & - & - & - & - & - \\
\hline GPC18 & F1839 & F2329 & F1440 & F2030 & F2109 & - & - & - & - & - & - & - \\
\hline GPC19 & F1839 & F2329 & F1440 & F2030 & F2109 & - & - & - & - & - & - & - \\
\hline GPC20 & F2265 & F2330 & F1560 & F1718 & - & - & - & - & - & - & - & - \\
\hline GPC21 & F1876 & F1675 & F1892 & F2006 & - & - & - & - & - & - & - & - \\
\hline GPC22 & F1298 & F1134 & F1394 & F1329 & F1072 & F853 & - & - & - & - & - & - \\
\hline GPC23 & F2136 & F2161 & F2193 & F2200 & - & - & - & - & - & - & - & - \\
\hline GPC24 & F1813 & F1638 & $\mathrm{F} 2315$ & F2277 & F2245 & F1581 & - & - & - & - & - & - \\
\hline GPC25 & F1994 & F1883 & $\mathrm{F} 2110$ & F2031 & F1937 & F1808 & - & - & - & - & - & - \\
\hline \multicolumn{13}{|c|}{ Prioritised features of WWTP influent samples in negative ionisation mode } \\
\hline GPC1 & F2846 & F2858 & F2893 & F2900 & F2962 & F3009 & F3015 & - & - & - & - & - \\
\hline GPC2 & F2390 & F2542 & F2681 & F2734 & F3103 & F3151 & F3202 & - & - & - & - & - \\
\hline GPC3 & F2745 & F2841 & F3003 & F3039 & F3040 & F3048 & - & - & - & - & - & - \\
\hline GPC4 & F2825 & F2837 & F2965 & F2973 & F3158 & F3264 & - & - & - & - & - & - \\
\hline GPC5 & F2400 & F2616 & F2864 & F2883 & F2958 & - & - & - & - & - & - & - \\
\hline GPC6 & F2706 & F2929 & F3063 & F3064 & - & - & - & - & - & - & - & - \\
\hline GPC7 & F2448 & F2531 & F2576 & - & - & - & - & - & - & - & - & - \\
\hline GPC8 & F2532 & F2755 & - & - & - & - & - & - & - & - & - & - \\
\hline
\end{tabular}


Table S- 12 - List of $\mathrm{m} / \mathrm{z}$, retention time as well as the trend of relevant prioritised features using GPCA of positive and negative ionisation mode (cf. Table S-9).

\begin{tabular}{|c|c|c|c|}
\hline Feature ID & Trend & $m / z$ & Retention time in min \\
\hline \multicolumn{4}{|c|}{ Prioritised features of positive ionisation mode } \\
\hline F1457 & decreasing & 281.1128 & 7.01 \\
\hline F1459 & decreasing & 281.1132 & 7.90 \\
\hline F1618 & decreasing & 313.1390 & 8.34 \\
\hline F1687 & decreasing & 335.1214 & 8.90 \\
\hline F1778 & decreasing & 363.1161 & 6.53 \\
\hline F1686 & decreasing & 335.1212 & 8.34 \\
\hline F1713 & decreasing & 341.1337 & 6.55 \\
\hline F1714 & decreasing & 341.1338 & 7.57 \\
\hline F1617 & decreasing & 313.1389 & 8.92 \\
\hline F1717 & decreasing & 343.1497 & 6.34 \\
\hline F1538 & decreasing & 299.1237 & 5.58 \\
\hline F924 & decreasing & 173.0209 & 4.54 \\
\hline F2062 & increasing & 476.3063 & 8.94 \\
\hline F1978 & increasing & 432.2800 & 8.36 \\
\hline F2131 & increasing & 520.3326 & 9.46 \\
\hline F1870 & increasing & 388.2535 & 7.72 \\
\hline F2223 & increasing & 564.3586 & 9.92 \\
\hline F1808 & increasing & 371.2275 & 7.71 \\
\hline F1937 & increasing & 415.2540 & 8.36 \\
\hline F1719 & increasing & 344.2275 & 6.97 \\
\hline F2031 & increasing & 459.2804 & 8.94 \\
\hline F2268 & increasing & 608.3848 & 10.34 \\
\hline F1612 & decreasing & 312.2532 & 17.91 \\
\hline F2121 & decreasing & 511.5189 & 19.06 \\
\hline F2145 & decreasing & 528.3640 & 11.61 \\
\hline F1935 & decreasing & 415.2538 & 6.91 \\
\hline F2243 & decreasing & 585.5196 & 19.06 \\
\hline F2166 & decreasing & 537.5346 & 19.21 \\
\hline F1616 & decreasing & 312.3260 & 20.74 \\
\hline F1747 & decreasing & 353.3158 & 16.99 \\
\hline F1809 & decreasing & 371.2275 & 6.21 \\
\hline F1581 & decreasing & 306.2400 & 17.91 \\
\hline F733 & decreasing & 153.0909 & 17.91 \\
\hline F913 & decreasing & 170.1175 & 17.89 \\
\hline F1232 & decreasing & 228.1953 & 19.72 \\
\hline F742 & decreasing & 154.1224 & 17.91 \\
\hline F916 & decreasing & 171.1015 & 18.15 \\
\hline F1429 & decreasing & 270.2786 & 19.54 \\
\hline F751 & decreasing & 155.1066 & 17.89 \\
\hline F728 & decreasing & 152.1067 & 17.90 \\
\hline F1286 & decreasing & 237.0866 & 10.02 \\
\hline F1214 & decreasing & 222.0760 & 5.58 \\
\hline F959 & decreasing & 178.0496 & 5.58 \\
\hline
\end{tabular}


Table S- 12 continued.

\begin{tabular}{|c|c|c|c|}
\hline F960 & decreasing & 178.0498 & 7.98 \\
\hline F1381 & decreasing & 259.0689 & 10.02 \\
\hline $\mathrm{F} 227$ & increasing & 100.0755 & 4.88 \\
\hline F1529 & increasing & 298.8861 & 1.12 \\
\hline F2225 & decreasing & 567.5820 & 20.00 \\
\hline F2155 & decreasing & 533.5019 & 19.05 \\
\hline F1716 & decreasing & 341.2428 & 10.65 \\
\hline F2147 & decreasing & 529.4617 & 22.21 \\
\hline F2185 & decreasing & 550.3464 & 11.63 \\
\hline F2245 & decreasing & 589.5643 & 20.02 \\
\hline F2169 & decreasing & 539.5505 & 19.99 \\
\hline F935 & decreasing & 175.0152 & 8.88 \\
\hline F1240 & decreasing & 231.0781 & 8.88 \\
\hline F1349 & decreasing & 251.0468 & 8.18 \\
\hline F1097 & decreasing & 198.1277 & 5.42 \\
\hline F192 & decreasing & 98.9840 & 2.12 \\
\hline F1020 & decreasing & 186.0858 & 3.67 \\
\hline F398 & decreasing & 125.0153 & 3.67 \\
\hline F1006 & decreasing & 184.0887 & 3.67 \\
\hline F92 & decreasing & 84.0557 & 2.20 \\
\hline F570 & decreasing & 137.0963 & 17.89 \\
\hline F1032 & decreasing & 186.1487 & 17.89 \\
\hline F1896 & decreasing & 399.3941 & 16.17 \\
\hline F1917 & decreasing & 408.3081 & 19.71 \\
\hline F1913 & decreasing & 407.3238 & 16.99 \\
\hline F1488 & decreasing & 286.3008 & 19.99 \\
\hline F1574 & decreasing & 304.2999 & 14.86 \\
\hline F1839 & decreasing & 380.2765 & 18.90 \\
\hline F2329 & decreasing & 680.4804 & 21.95 \\
\hline F1440 & decreasing & 276.1443 & 16.44 \\
\hline F2030 & decreasing & 459.2802 & 7.48 \\
\hline F2109 & decreasing & 503.3064 & 8.02 \\
\hline F1876 & decreasing & 389.3372 & 17.93 \\
\hline F1675 & decreasing & 330.2641 & 17.91 \\
\hline F1892 & decreasing & 398.2144 & 16.43 \\
\hline F2006 & decreasing & 445.4000 & 19.74 \\
\hline F1298 & decreasing & 240.1573 & 9.53 \\
\hline F1134 & decreasing & 204.9793 & 2.03 \\
\hline F1394 & decreasing & 263.0211 & 2.03 \\
\hline F1329 & decreasing & 247.2415 & 20.56 \\
\hline F853 & decreasing & 165.1122 & 17.89 \\
\hline F2136 & increasing & 523.0462 & 5.27 \\
\hline F2161 & increasing & 537.0352 & 5.26 \\
\hline F2193 & increasing & 552.0001 & 5.27 \\
\hline F2200 & increasing & 553.0063 & 5.26 \\
\hline \multicolumn{4}{|c|}{ Prioritised features of negative ionisation mode } \\
\hline F2846 & decreasing & 216.0328 & 12.86 \\
\hline
\end{tabular}


Table S- 12 continued.

\begin{tabular}{|c|c|c|c|}
\hline F2858 & decreasing & 218.0301 & 12.84 \\
\hline F2893 & decreasing & 232.0277 & 12.70 \\
\hline F2900 & decreasing & 234.0251 & 12.71 \\
\hline F2962 & decreasing & 249.9941 & 12.78 \\
\hline F3009 & decreasing & 269.9033 & 6.29 \\
\hline F3015 & decreasing & 271.9005 & 6.29 \\
\hline F2390 & decreasing & 77.0396 & 3.53 \\
\hline F2542 & decreasing & 121.0293 & 3.54 \\
\hline F2681 & decreasing & 165.0189 & 3.53 \\
\hline F2734 & decreasing & 179.0346 & 5.85 \\
\hline F3103 & decreasing & 321.2193 & 18.76 \\
\hline F3151 & decreasing & 353.0278 & 3.54 \\
\hline F3202 & decreasing & 383.9581 & 3.53 \\
\hline F2745 & decreasing & 187.0509 & 4.33 \\
\hline F2841 & decreasing & 214.0441 & 9.19 \\
\hline F3003 & decreasing & 269.0232 & 4.32 \\
\hline F3039 & decreasing & 293.1783 & 18.91 \\
\hline F3040 & decreasing & 294.0002 & 9.15 \\
\hline F3048 & decreasing & 295.9976 & 9.16 \\
\hline F2825 & decreasing & 209.0942 & 10.08 \\
\hline F2837 & decreasing & 212.9862 & 2.19 \\
\hline F2965 & decreasing & 250.9260 & 10.90 \\
\hline F2973 & decreasing & 252.9234 & 10.95 \\
\hline F3158 & decreasing & 353.2002 & 18.15 \\
\hline F3264 & decreasing & 529.4613 & 23.67 \\
\hline F2400 & decreasing & 78.9587 & 2.09 \\
\hline F2616 & decreasing & 153.0318 & 2.11 \\
\hline F2864 & decreasing & 221.1180 & 11.29 \\
\hline F2883 & decreasing & 229.0626 & 8.84 \\
\hline F2958 & decreasing & 249.0316 & 8.14 \\
\hline F2706 & decreasing & 171.0115 & 2.72 \\
\hline F2929 & decreasing & 243.0692 & 11.34 \\
\hline F3063 & decreasing & 299.1317 & 13.35 \\
\hline F3064 & decreasing & 299.1320 & 10.98 \\
\hline F2448 & decreasing & 93.0344 & 6.08 \\
\hline F2531 & decreasing & 118.0296 & 5.55 \\
\hline F2576 & decreasing & 137.0242 & 6.10 \\
\hline F2532 & decreasing & 118.0297 & 4.38 \\
\hline F2755 & decreasing & 193.0997 & 12.25 \\
\hline
\end{tabular}




\subsection{GPCA Results of Second Prioritisation Route}
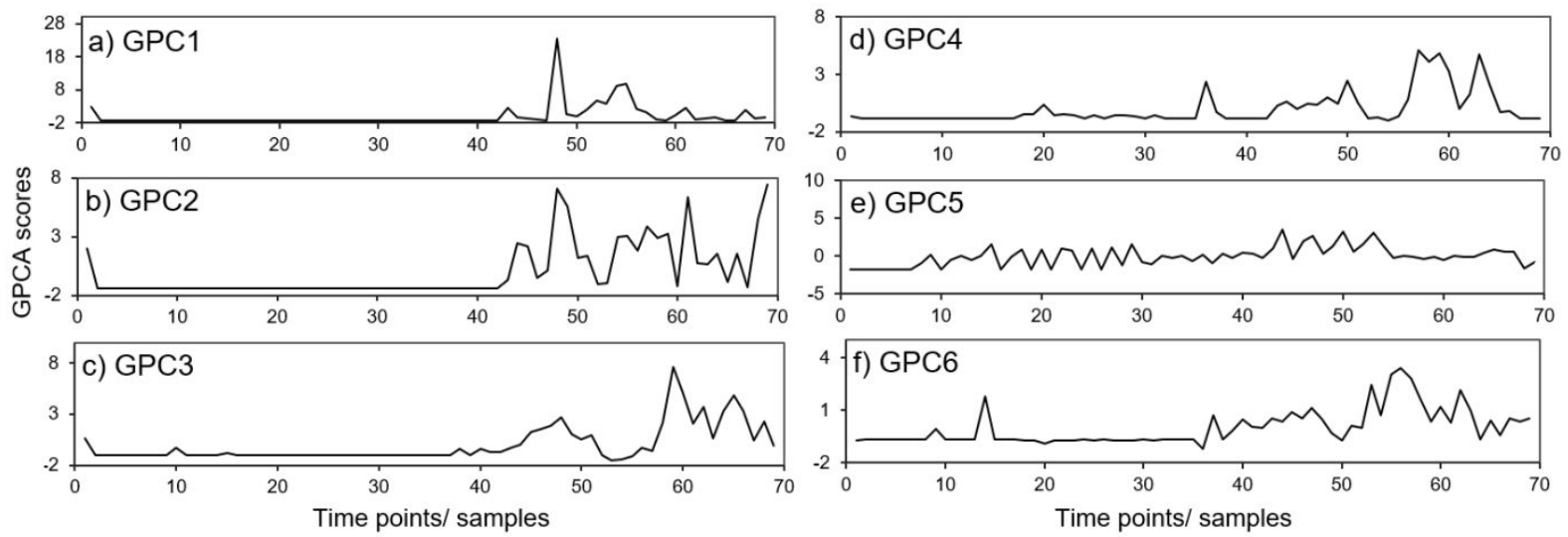

Figure S- 13 - Score vectors of six components from the GPCA model for the pre-filtered WWTP influent data matrix for positive ionisation mode. The prioritisation order is GPC6, GPC3, GPC1, GPC2, GPC5 and GPC4, respectively.

Table S- 13 - GPCA results for feature prioritisation of WWTP influent samples containing the whole subset of continuously increasing trends of positive ionisation mode.

\begin{tabular}{|lcllllllll|}
\hline GPC & Rank $^{*}$ & \multicolumn{10}{c|}{ Features in groups } \\
\hline GPC1 & 3 & F1883 & F1994 & F2110 & F2031 & F1937 & F1808 & F1719 & F1474 \\
GPC2 & 4 & F2200 & F2193 & F2136 & F2161 & F1805 & F1756 & & \\
GPC3 & 2 & F1756 & F1549 & F1696 & F1468 & F1561 & & & \\
GPC4 & 6 & F1857 & F1954 & F1529 & & & & & \\
GPC5 & 5 & F1182 & F188 & & & & & & \\
GPC6 & 1 & F1529 & F227 & & & & & & \\
\hline
\end{tabular}

*Ranking of GPCs performed using Spearman's rank correlation values.

\section{REFERENCES}

(1) Camacho, J.; Garcia-Teodoro, P.; Macia-Fernandez, G. Traffic Monitoring and Diagnosis with Multivariate Statistical Network Monitoring: A Case Study. Proc. - 2017 IEEE Symp. Secur. Priv. Work. SPW 2017 2017, 2017-Decem, 241-246. https://doi.org/10.1109/SPW.2017.11.

(2) Camacho, J.; García-Giménez, J. M.; Fuentes-García, N. M.; Maciá-Fernández, G. Multivariate Big Data Analysis for Intrusion Detection: 5 Steps from the Haystack to the Needle. Comput. Secur. 2019, 87, 101603. https://doi.org/https://doi.org/10.1016/j.cose.2019.101603.

(3) Camacho, J.; Theron, R.; Garcia-Gimenez, J. M.; Macia-Fernandez, G.; Garcia-Teodoro, P. Group-Wise Principal Component Analysis for Exploratory Intrusion Detection. IEEE Access 2019, 7, 113081-113093. https://doi.org/10.1109/access.2019.2935154.

(4) Camacho, J.; Rodríguez-Gómez, R. A.; Saccenti, E. Group-Wise Principal Component Analysis for Exploratory Data Analysis. J. Comput. Graph. Stat. 2017, 26 (3), 501-512. https://doi.org/10.1080/10618600.2016.1265527.

(5) Jollife, I. T. Principal Component Analysis; Springer-Verlag New York: New York, 2002. 
https://doi.org/https://doi.org/10.1007/b98835.

(6) Jolliffe, I. T.; Trendafilov, N. T.; Uddin, M. A Modified Principal Component Technique Based on the LASSO. J. Comput. Graph. Stat. 2003, 12 (3), 531-547. https://doi.org/10.1198/1061860032148.

(7) Camacho, J. Observation-Based Missing Data Methods for Exploratory Data Analysis to Unveil the Connection between Observations and Variables in Latent Subspace Models. J. Chemom. 2011, 25 (11), 592-600. https://doi.org/10.1002/cem.1405.

(8) Nelson, P. R. C.; Taylor, P. A.; MacGregor, J. F. Missing Data Methods in PCA and PLS: Score Calculations with Incomplete Observations. Chemom. Intell. Lab. Syst. 1996. https://doi.org/10.1016/S0169-7439(96)00007-X.

(9) Lamchin, M.; Lee, W. K.; Jeon, S. W.; Wang, S. W.; Lim, C. H.; Song, C.; Sung, M. Corrigendum to "Mann-Kendall Monotonic Trend Test and Correlation Analysis Using Spatio-Temporal Dataset: The Case of Asia Using Vegetation Greenness and Climate Factors" (MethodsX (2018) 5 (803-807), (S2215016118301134), (10.1016/j.Mex.2018.07.006)). MethodsX 2019, 6, 1379 1383. https://doi.org/10.1016/j.mex.2019.05.030.

(10) Mondal, A.; Kundu, S.; Mukhopadhyay, A. 70 Rainfall Trend Analysis by Mann-Kendall Test: A Case Study of North-Eastern Part of Cuttack District, Orissa. Online) An Online Int. J. Available 2012, 2 (1), 70-78.

(11) Sharma, D.; Kumar, B.; Chand, S. A Trend Analysis of Machine Learning Research with Topic Models and Mann-Kendall Test. Int. J. Intell. Syst. Appl. 2019, 11 (2), 70-82. https://doi.org/10.5815/ijisa.2019.02.08.

(12) Shadmani, M.; Marofi, S.; Roknian, M. Trend Analysis in Reference Evapotranspiration Using Mann-Kendall and Spearman's Rho Tests in Arid Regions of Iran. Water Resour. Manag. 2012, 26 (1), 211-224. https://doi.org/10.1007/s11269-011-9913-z.

(13) Ahmad, I.; Tang, D.; Wang, T.; Wang, M.; Wagan, B. Precipitation Trends over Time Using MannKendall and Spearman's Rho Tests in Swat River Basin, Pakistan. Adv. Meteorol. 2015, 2015. https://doi.org/10.1155/2015/431860.

(14) Yue, S.; Pilon, P.; Cavadias, G. Power of the Mann-Kendall and Spearman's Rho Tests for Detecting Monotonic Trends in Hydrological Series. J. Hydrol. 2002, 259 (1), 254-271. https://doi.org/https://doi.org/10.1016/S0022-1694(01)00594-7. 